Chapter published in "Advanced Semiconductors and Organic

Nano-Techniques", edited by H. Morkoc, Academic Press 2003

\title{
Electrical Conduction through Molecules
}

\author{
Ferdows Zahid, Magnus Paulsson and Supriyo Datta* \\ School Of Electrical \& Computer Engineering, \\ Purdue University, \\ 1285 Electrical Engineering Building, \\ West Lafayette, IN - 47907-1285, USA
}

1 Introduction

July 8, 2003

2 Molecular Conduction: Qualitative Picture 4

2.1 Energy Level Diagram . . . . . . . . . . . . . . . 4

2.2 Broadening by the contacts . . . . . . . . . . . . 7

2.3 Potential Profile . . . . . . . . . . . . . . 8

3 Toy Models 11

3.1 Discrete Level Model . . . . . . . . . . . . . . . . . . . . . . . . . 11

3.2 Model with Broadening . . . . . . . . . . . . . . . . 15

3.3 Unrestricted Model . . . . . . . . . . . . . . . . . . . . . 15

4 A Realistic Multilevel Model $\quad 17$

4.1 Non-Equilibrium Green's Function (NEGF) Formalism . . . . . . 17

4.2 Hamiltonian . . . . . . . . . . . . . . . . . . . . 20

4.3 Self Energy . . . . . . . . . . . . . . . . . . . . . 22

4.4 Self-Consistent Potential . . . . . . . . . . . . . . . . . . 24

5 Application of the NEGF Formalism 25

5.1 Gold Contacts . . . . . . . . . . . . . . . . 26

5.2 Phenyl Dithiol . . . . . . . . . . . . . . . . . . . 27

5.2 .1 Equilibrium Properties . . . . . . . . . . . . . . . 28

5.2 .2 Non Equilibrium Properties . . . . . . . . . . . . . 30

5.2 .3 Asymmetric Coupling to the Contacts . . . . . . . . . 31

6 Summary and Conclusions 32

A NEGF in Non-Orthogonal Basis 33

B Surface Green's Function $\quad 35$

*datta@purdue.edu 
Electrical Conduction through Molecules

\section{Introduction}

In recent years, several experimental groups have reported measurements of the current-voltage (I-V) characteristics of individual or small numbers of molecules. Even three-terminal measurements showing evidence of transistor action has been reported using carbon nanotubes $[1,2]$ as well as self-assembled monolayers of conjugated polymers. These developments have attracted much attention from the semiconductor industry who are actively looking for ways to progress from gigabit to terabit integration by complementing or even replacing presentday CMOS circuitry. There is great interest therefore from an applied point of view to model and understand the capabilities of molecular conductors. At the same time, this is also a topic of great interest from the point of view of basic physics. A molecule represents a quantum dot, at least an order of magnitude smaller than semiconductor quantum dots, which allows us to study many of the same mesoscopic and/or many-body effects at far higher temperatures.
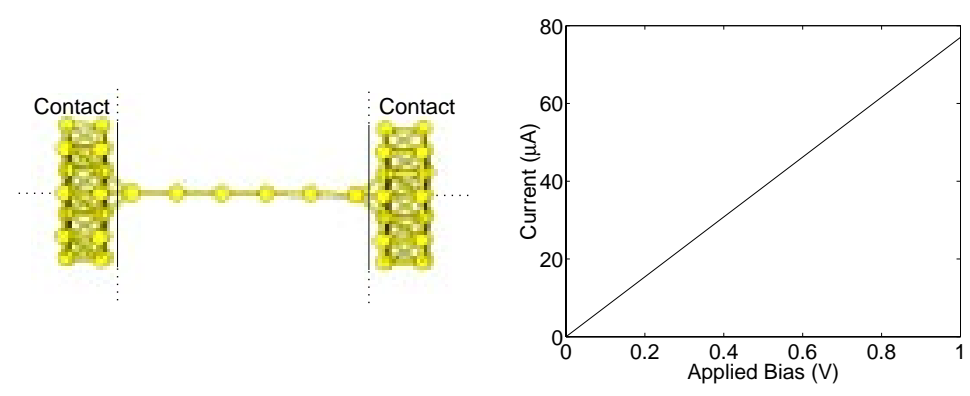

Figure 1: Left, wire consisting of six gold atoms forming a Quantum Point Contact (QPC). Right, quantized conductance $\left(I=\frac{e^{2}}{\pi \hbar} V\right)$.

Consider for example a gold wire stretched between two gold surfaces as shown in Fig. 1. One of the seminal results of mesoscopic physics is that such a wire has a quantized conductance equal to $\frac{e^{2}}{\pi \hbar} \sim 77.5 \mu \mathrm{A} / \mathrm{V} \sim(12.9 \mathrm{k} \Omega)^{-1}$. This was first established using semiconductor structures $[3,4,5]$ at $4 \mathrm{~K}$, but recent experiments on gold contacts have demonstrated it at room temperature [6]. How can a wire have a resistance that is independent of its length? The answer is that this resistance is really associated with the interfaces between the narrow wire and the wide contacts. If there is scattering inside the wire it would give rise to an additional resistance in series with this fundamental interface resistance. The fact that a short wire has a resistance of $12.9 \mathrm{k} \Omega$ is a non-obvious result that was not known before 1988 .

What happens if we replace the gold wire with a molecular wire? Most commonly we get I-V characteristics of the type sketched in Fig. 2. This has been observed using many different approaches including breakjunctions $[7,8$, $9,10,11]$, scanning probes $[12,13,14,15]$, nanopores [16] and a host of other methods (see for example [17]). A number of theoretical models have been 

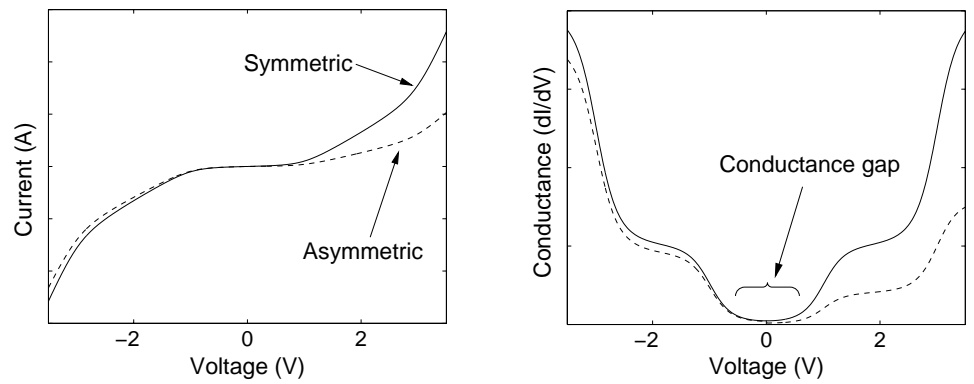

Figure 2: Schematic picture, showing general properties of measured currentvoltage (I-V) and conductance $(\mathrm{G}-\mathrm{V})$ characteristics for molecular wires. Solid line, symmetrical I-V. Dashed line, asymmetrical I-V.

developed for calculating the I-V characteristics of molecular wires using semiempirical $[15,18,19,20,21]$ as well as first principles $[22,23,24,25,26,27,28]$ theory.

In sections 2 and 3 we will show that the basic features of these observed $\mathrm{I}-\mathrm{V}$ characteristics are easily understood in terms of three factors: (1) Distance $\left|E_{f}-\epsilon_{0}\right|$ from the Fermi energy $E_{f}$ to the nearest molecular level $\epsilon_{0},(2)$ Broadening $\Gamma_{1}, \Gamma_{2}$ of the molecular levels due to the coupling to the contacts and (3) the charging energy $U$ per electron. We will present simple toy models to illustrate the role of these three factors in determining the shape of the I-V characteristics. These observations are supported by more detailed treatments, both semi-empirical and ab initio, but the toy models make them more obvious. In particular there is one point that we would like to stress. It is common in the literature to associate the conductance gap with the gap between the highest occupied molecular orbital (HOMO) and the lowest unoccupied molecular orbital (LUMO) and we often see statements like "conductance measurements show a HOMO-LUMO gap of $2.5 \mathrm{eV}$ ". However, we believe that the conductance gap is related to the distance from the Fermi energy $E_{f}$ to the nearest molecular energy level $\epsilon_{0}$. Indeed that is what makes transistor action possible: the gate electrode basically moves the molecular level $\epsilon_{0}$ relative to the Fermi energy $E_{f}$. However, this also makes the conductance gap depend sensitively on the surface conditions and can vary widely from one theoretical model to another, depending on the specific assumptions made. Even with the same metal, the precise location of $E_{f}$ (and hence the conduction gap) can depend sensitively on the surface conditions as is well known from the long-standing controversies over the location of $E_{f}$ at metal-semiconductor interfaces. As such we believe that more theoretical and experimental effort should be focused on establishing the precise location of $E_{f}$ for different metal-molecule "hetrostructures" with well characterized interfaces.

The general shape of the I-V characteristics of molecular conductors commonly resembles those shown in Fig. 2. However, what makes the field of 
Electrical Conduction through Molecules

molecular electronics particularly exciting is the possibility of engineering novel molecules that exhibit all kinds of I-V characteristics beyond those shown in Fig. 2. For example, there is evidence for special molecules that exhibit switching behavior [16] although the precise mechanism is still unclear. In section 4 we present a rigorous transport formalism, the Non-Equilibrium Green's Function (NEGF) formalism, that can be used in conjunction with a suitable molecular Hamiltonian (semi-empirical or ab initio) to investigate the I-V characteristics of different molecules. Illustrative examples are presented in section 5 using a Hückel Hamiltonian and a simple charging model, but the same basic approach can be used to combine the NEGF transport formalism with more sophisticated ab initio approaches to electronic structure calculations as discussed in the related articles [29, 30]. The simple model described in section 5 is publicly available through the Purdue Simulation Hub ("www.nanohub.purdue.edu") and can be run without any need for installation. This is an improved version of the earlier model [15] made available in 1999. Further improvements may be needed to take into account the role of inelastic scattering or polaronic effects especially in larger molecules like DNA chains or long polymers.

\section{Molecular Conduction: Qualitative Picture}

To understand the flow of current through molecules, we need three basic ingredients: (1) An energy level diagram showing the molecular energy levels relative to the Fermi energy in the metallic contacts, (2) an estimate of the broadening of the molecular levels due to the coupling to the contacts and (3) the spatial profile of the applied potential under bias. Let us discuss these issues one by one.

\subsection{Energy Level Diagram}

The first step in understanding the current (I) vs. voltage (V) curve for a molecular conductor is to draw an energy level diagram and locate the Fermi energy. Consider first a molecule sandwiched between two metallic contacts, but with very weak electronic coupling. We could then line up the energy levels as shown in Fig. 3 using the metallic work function (WF) and the electronic affinity $(E A)$ and ionization potential $(I P)$ of the molecule. For example, a (111) gold surface has a work function of $\sim 5.3 \mathrm{eV}$ while the electron affinity and ionization potential, $E A_{0}$ and $I P_{0}$, for isolated phenyl dithiol ${ }^{1}$ in the gas phase have been reported to be $\sim 2.4 \mathrm{eV}$ and $8.3 \mathrm{eV}$ respectively [31]. These values are associated with electron emission and injection to and from a vacuum and may need some modification to account for the metallic contacts. For example the actual $E A, I P$ will possibly be modified from $E A_{0}, I P_{0}$ due to the image potential $W_{i m}$ associated with the metallic contacts [32]:

$$
E A=E A_{0}+W_{i m}
$$

\footnotetext{
${ }^{1} \mathrm{~A}$ schematic picture of this conjugated molecule is shown in Fig. 17. We will use it as an example throughout the chapter.
} 


$$
I P=I P_{0}-W_{i m}
$$
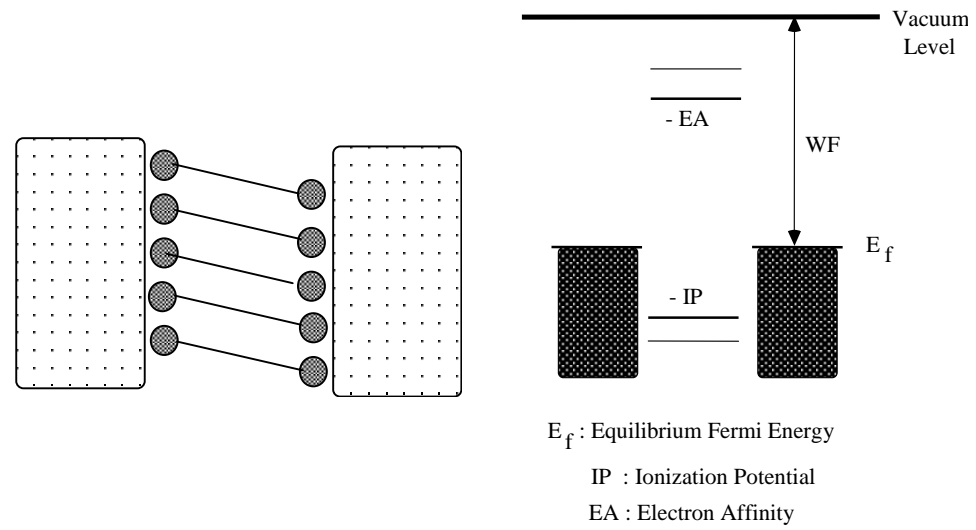

Figure 3: Equilibrium energy level diagram for a metal-molecule-metal sandwich for a weakly coupled molecule.

The probability of the molecule losing an electron to form a positive ion is equal to $e^{(W F-I P) / k_{B} T}$ while the probability of the molecule gaining an electron to form a negative ion is equal to $e^{(E A-W F) / k_{B} T}$. We thus expect the molecule to remain neutral as long as both $(W F-I P)$ and $(E A-W F)$ are much larger than $k_{B} T$, a condition that is usually satisfied for most metal-molecule combinations. Since it costs too much energy to transfer one electron into or out of the molecule, it prefers to remain neutral in equilibrium.

The picture changes qualitatively if the molecule is chemisorbed directly on the metallic contact, see Fig. 4. Then the molecular energy levels are broadened significantly by the strong hybridization with the delocalized metallic wavefunctions, making it possible to transfer fractional amounts of charge to or from the molecule. Indeed there is a change in the electrostatic potential inside the molecule due to the charge transfer and the energy levels of the molecule are shifted by a contact potential (CP), see Fig. 4 .

It is now more appropriate to describe transport in terms of the HOMOLUMO levels associated with incremental charge transfer [33] rather than the affinity and ionization levels associated with integer charge transfer. Whether the molecule-metal coupling is strong enough for this to occur depends on the relative magnitudes of the single electron charging energy $(U)$ and energy level broadening $(\Gamma)$. As a rule of thumb, if $U>>\Gamma$, we can expect the structure to be in the Coulomb Blockade $(\mathrm{CB})$ regime characterized by integer charge transfer; otherwise it is in the self-consistent field $(\mathrm{SCF})$ regime characterized by fractional charge transfer. This is basically the same criterion that one uses for the Mott transition in periodic structures, with $\Gamma$ playing the role of the hopping matrix element. It is important to note that for a structure to be in the $\mathrm{CB}$ regime both contacts must be weakly coupled, since the total broadening 

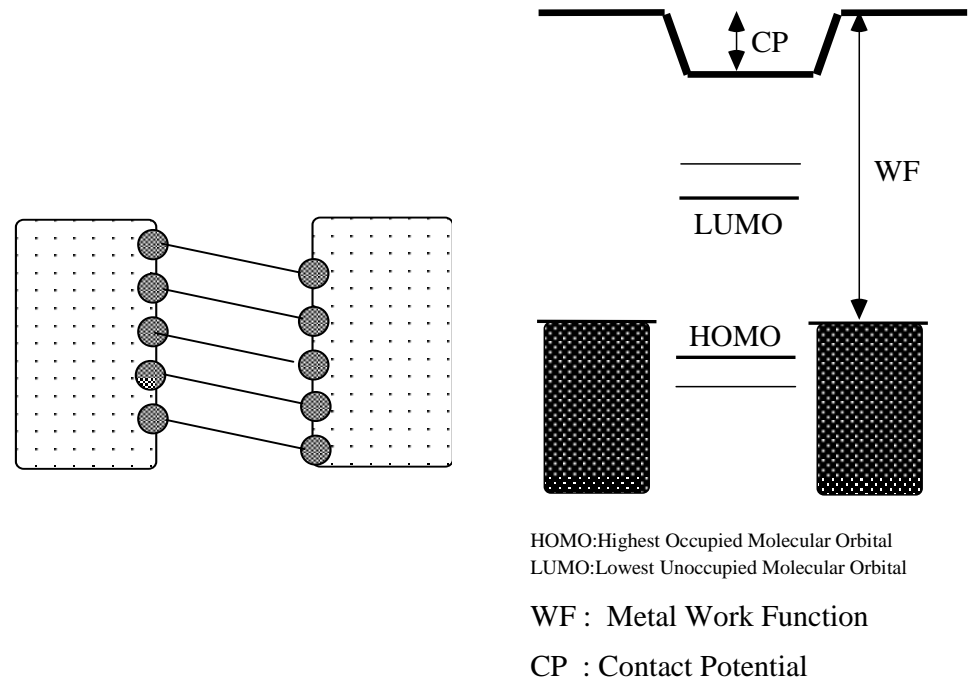

Figure 4: Equilibrium energy level diagram for a metal-molecule-metal sandwich for a molecule strongly coupled to the contacts.

$\Gamma$ is the sum of the individual broadening due to the two contacts. Even if only one of the contacts is coupled strongly we can expect $\Gamma \sim U$ thus putting the structure in the SCF regime. See section 3.3 and Fig. 15 for a toy model illustration of the I-V characteristics in the $\mathrm{CB}$ regime.

Where is the Fermi energy? The location of the Fermi energy relative to the HOMO and LUMO levels is probably the most important factor in determining the current (I) versus voltage (V) characteristics of molecular conductors. Usually it lies somewhere inside the HOMO-LUMO gap. To see this, we first note that $E_{f}$ is located by the requirement that the number of states below the Fermi energy must be equal to the number of electrons in the molecule. But this number need not be equal to the integer number we expect for a neutral molecule. A molecule does not remain exactly neutral when connected to the contacts. It can and does pick up a fractional charge depending on the work function of the metal. However, the charge transferred $(\delta n)$ for most metal-molecule combinations is usually much less than one. If $\delta n$ were equal to +1 , the Fermi energy would lie on the LUMO while if $\delta n$ were -1 , it would lie on the HOMO. Clearly for values in between, it should lie somewhere in the HOMO-LUMO gap.

To estimate the amount of charge transfered it is useful to introduce the concept of a charge neutrality level which denotes the location of the Fermi energy for a neutral molecule (CNL) $[34,35]$. We use $E_{N}^{0}$ and $E_{N}$ to denote its location before and after charge transfer has taken place, see Fig. 5 . We can write:

$$
\delta n=D\left(E_{f}-E_{N}\right)
$$




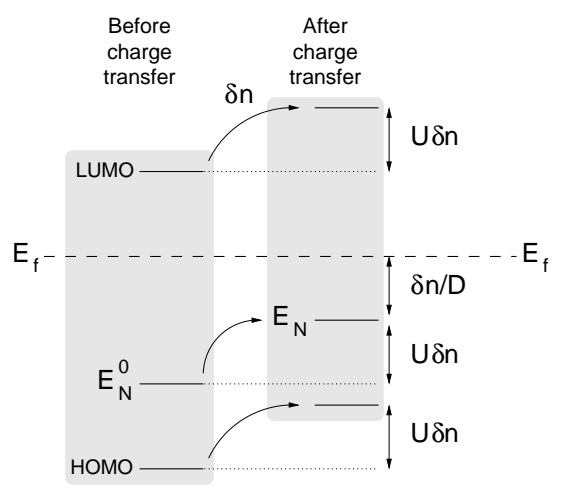

Figure 5: Position of the charge neutrality level before $\left(E_{N}^{0}\right)$ and after charge transfer $\left(E_{N}\right)$ relative to the contact Fermi energy.

$$
E_{N}=E_{N}^{0}+U \delta n
$$

where $U$ is the charging energy per electron and $D$ is the density of states (assumed to be constant over this energy range for simplicity). From Eqs. 3, 4:

$$
\begin{aligned}
\delta n & =\left(E_{f}-E_{N}^{0}\right) \frac{D}{1+U D} \\
E_{f}-E_{N} & =\frac{E_{f}-E_{N}^{0}}{1+U D}
\end{aligned}
$$

This simple argument shows that the amount of charge transfer is driven by the difference $\left(E_{f}-E_{N}^{0}\right)$ between the contact Fermi energy and the neutrality level of the molecule (before charge transfer). But if the factor $U D$ is large, the neutrality level will align with $E_{f}$ after charge transfer has taken place.

A number of authors have performed detailed calculations to locate the Fermi energy with respect to the molecular levels for a phenyl dithiol molecule sandwiched between gold contacts, but there is considerable disagreement. Different theoretical groups have placed it close to the LUMO [19, 24] or to the HOMO $[15,22]$. As noted in the introduction, the density of states inside the HOMOLUMO gap is quite small making the precise location of the Fermi energy very sensitive to small amounts of electron transfer, a fact that could have a significant effect on both theory and experiment. As such it seems justifiable to treat $E_{f}$ as a "fitting parameter" within reasonable limits when trying to explain experimental I-V curves.

\section{$2.2 \quad$ Broadening by the contacts}

It is evident that the strength of coupling of the molecule to the contacts is important in determining the current flow - the stronger the coupling, the larger 


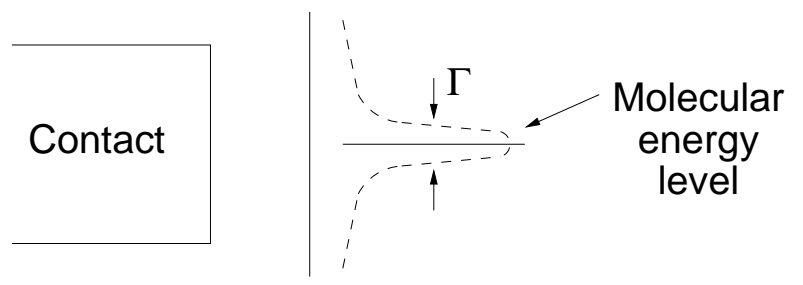

Figure 6: Energy level broadening.

the current. A useful quantitative measure of the coupling is the resulting broadening $\Gamma$ of the molecular energy levels, see Fig. 6 .

This broadening $\Gamma$ can also be related to the time $\tau$ it takes for an electron placed in that level to escape into the contact: $\Gamma=\hbar / \tau$. One could also interpret $\Gamma / \hbar$ as the rate at which electrons are injected into the level from the contact. In general, the broadening $\Gamma$ could be different for different energy levels. Also it is convenient to define two quantities $\Gamma_{1}$ and $\Gamma_{2}$, one for each contact, with the total broadening $\Gamma=\Gamma_{1}+\Gamma_{2}$.

\subsection{Potential Profile}

A very important factor in determining the I-V characteristics is the voltage profile across the conductor. At equilibrium, the entire system has a common Fermi energy $E_{f}$ which is equal to the electrochemical potentials $\mu_{1}$ and $\mu_{2}$ in the two contacts. When we apply a voltage $V_{a p p l}$ across the structure we cause $\mu_{1}$ and $\mu_{2}$ to split by $e V_{a p p l}: \mu_{1}-\mu_{2}=e V_{a p p l}$. But how are $\mu_{1}$ and $\mu_{2}$ disposed with respect to the molecular levels?

We are of course free to choose any reference for the zero of our applied potential. For example, we could take contact 1 as our reference and write:

$$
\mu_{1}=E_{f} \quad: \quad \mu_{2}=E_{f}+e V_{a p p l}
$$

But we also have to take into account the shifting of the molecular levels, which depends on the detailed shape of the potential profile inside the molecule. To a lowest order approximation, we could say that the molecular levels shift "rigidly" [36] by the change in the average potential, $\left\langle\delta v_{m o l}(r)\right\rangle$ inside the molecule due to the applied bias.

Let us denote this average potential as:

$$
\left\langle\delta v_{m o l}(r)\right\rangle=\eta e V_{a p p l}
$$

where the voltage division factor $\eta$ is a number between 0 and 1 . It is often convenient to take the molecular levels as our reference, and instead shift the chemical potentials by:

$$
\mu_{1}=E_{f}-\eta e V_{a p p l} \quad: \quad \mu_{2}=E_{f}+(1-\eta) e V_{a p p l}
$$


(a) $\eta=0$ : Molecular levels remain fixed with respect to contact 1.
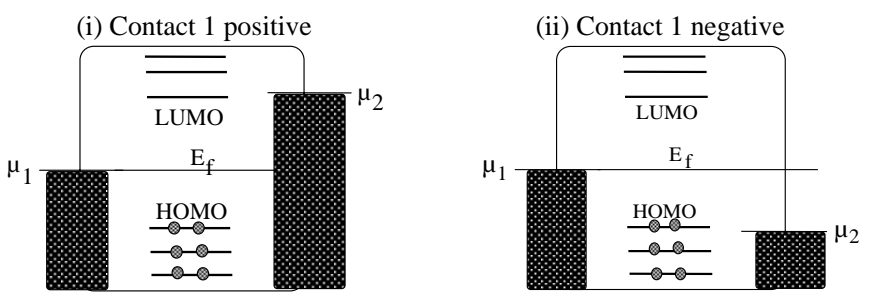

(b) $\eta=0.5$ : Molecular levels shift with respect to contact 1 by half the applied bias.
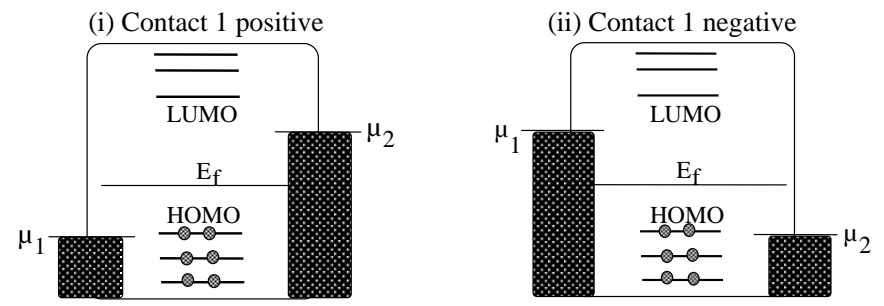

Figure 7: Schematic energy level diagram of metal-molecule-metal structure when contact 1 is (i) positively biased and when contact 1 is (ii) negatively biased with respect to contact 2. (a) $\eta=0$ : Molecular energy levels remain fixed with respect to contact 1 , and (b) $\eta=0.5$ : Molecular energy levels shift by half the applied voltage, with respect to contact 1 .

It is important to note that this voltage division factor, $\eta$ can have a profound effect on the I-V characteristics. If $\eta=0$, then the energy level diagram under bias looks as shown in Fig. 7(a). For a positive bias applied to contact 1, current starts to flow when, $\mu_{2}$ crosses the LUMO, while for negative bias, current starts to flow when $\mu_{2}$ crosses the HOMO. Thus, the positive branch of the I-V characteristics can look very different from the negative branch, since they involve different molecular orbitals. By contrast, if $\eta=0.5$, then the energy level diagram under bias looks as shown in Fig. 7(b). For either bias polarity, conduction takes place through the HOMO and the LUMO plays no role at all, assuming that the equilibrium Fermi energy $E_{f}$ is much closer to the HOMO to start with. If $E_{f}$ is much closer to the LUMO, then conduction will take place through the LUMO and the HOMO will play no role. In either case the $\mathrm{I}-\mathrm{V}$ characteristics will look much more symmetric, since the same orbitals are involved for either bias direction.

The voltage division factor, $\eta$ is basically a one-parameter characterization of the profile of the applied potential inside the molecule as illustrated in Fig. 8 with four idealized examples. To understand how these different profiles can arise, we note that in general the actual potential profile can be written in the 
form:

$$
\delta v_{m o l}(r)=v_{a p p l}(r)+F[\delta \rho(r)]
$$

where $\mathrm{F}$ represents an appropriate functional of the change in the charge density, $\delta \rho$ within the molecule and $v_{a p p l}(r)$ is the external potential due to the voltage applied between the contacts. For low voltages (less than the conductance gap) there is very little change in the charge density because the molecule has a very low density of states within the gap ${ }^{2}$; consequently $\delta v_{m o l}(r) \sim v_{a p p l}(r)$, where $v_{\text {appl }}$ is determined from simple electrostatics by solving the Laplace equation $(\epsilon$ : dielectric constant):

$$
\bar{\nabla} \cdot\left(\epsilon \bar{\nabla} v_{a p p l}(r)\right)=0
$$
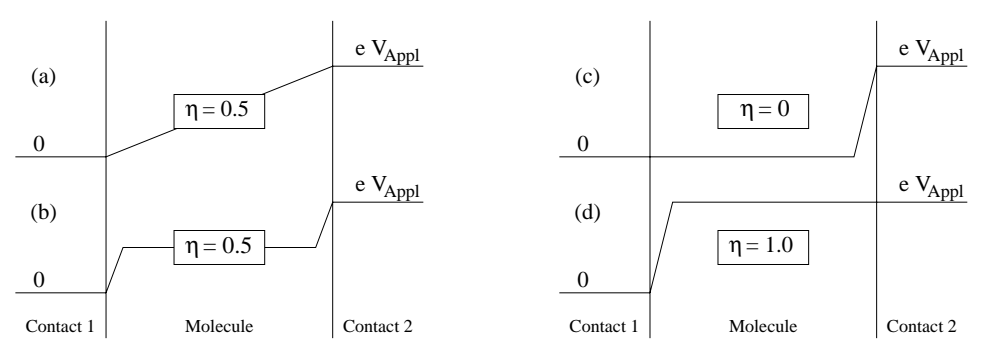

Figure 8: Four examples of potential profiles, illustrating the meaning of the voltage division factor $\eta$.

If the contact area is large compared to the length of the molecule, then we can solve a one-dimensional Laplace equation:

$$
\frac{\partial^{2} v_{a p p l}}{\partial z^{2}}=0
$$

which yields a linear potential profile a shown in Fig. 8(a) and $\eta=0.5$ if the molecule is roughly halfway between the contacts. If the molecule is highly polarizable (large $\epsilon$ ) then the potential profile could be flat inside the molecule (Fig. 8(b)), again with $\eta=0.5$. In any case, the coupling to the contacts has very little effect on $\delta v_{m o l}$ and hence on $\eta$. But if there is significant density of states in the molecule then it will lose or gain charge depending on the relative coupling to the contacts and the resulting potential profile will change to (c) or (d) depending on the relative coupling to contacts 1 and 2 (Fig. 8(c, d)). For this reason, although $\eta=0.5$ is appropriate at low bias the effective $\eta$ could change with bias as the molecule starts to conduct, as noted in Ref. [15]. In this chapter, we will use $\eta=0.5$ and account for charging effects explicitly with a simple model.

\footnotetext{
${ }^{2}$ This may not be true if the contacts are very strongly coupled so that there is a significant density of Metal-Induced-Gap States (MIGS). In this case the potential profile would be determined by the requirement of charge neutrality. For equal coupling to the two contacts the potential would look like Fig. 8(b) or for unequal coupling Fig. 8(c/d).
} 


\section{$3 \quad$ Toy Models}

In this section we will introduce a toy model with just one molecular energy level $\epsilon$. This level could represent either the HOMO or the LUMO whichever is believed to be closer to the equilibrium Fermi energy $E_{f}$. This simple one-level model incorporates the essential factors described in Section 2, and leads to realistic I-V plots that illustrate the roles played by each of these factors: (1) location of $E_{f}$ with respect to $\epsilon,(2)$ broadenings $\Gamma_{1}, \Gamma_{2}$ due to the two contacts and (3) charging energy $U$ which affects the shift in molecular energy levels under bias.

\subsection{Discrete Level Model}

A useful toy model is easily set up with one molecular level $\epsilon$ if we neglect the broadening and treat it as discrete. The current through the level can then be found from a simple sequential picture, see Fig. 9. Let $\Gamma_{1}\left(\Gamma_{2}\right)$ denote the coupling between the left (right) contact and the level. As discussed in section 2.2 , the rate at which an electron can escape into the left (right) contact is then $\Gamma_{1} / \hbar\left(\Gamma_{2} / \hbar\right)$.

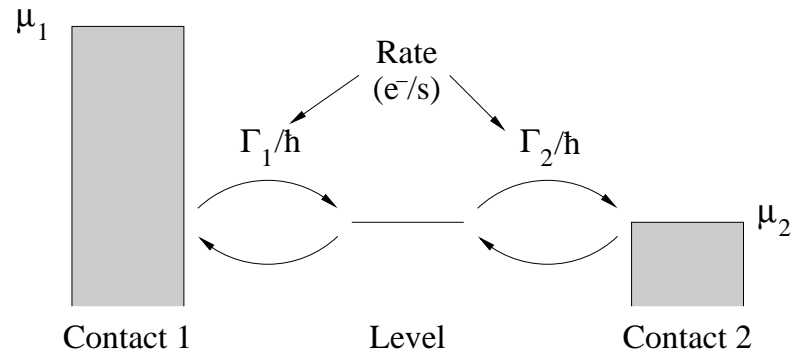

Figure 9: Illustration of the sequential model, i.e., similar to a reservoir being filled from one side and emptied into both sides.

If the level were in equilibrium with contact 1 then the number of electrons $N_{1}$, occupying the level would be given by:

$$
N_{1}=2(\text { for } \operatorname{spin}) f\left(\epsilon, \mu_{1}\right)
$$

while if it were in equilibrium with contact 2 the number would be:

$$
N_{2}=2(\text { for } \operatorname{spin}) f\left(\epsilon, \mu_{2}\right)
$$

where:

$$
f(\epsilon, \mu)=\left(1+e^{\frac{\epsilon-\mu}{k_{B} T}}\right)^{-1}
$$

is the Fermi-Dirac function. Under non-equilibrium conditions the number of electrons $N$ will be somewhere in between $N_{1}$ and $N_{2}$ and we can write the net 
current at the left junction as:

$$
I_{L}=\frac{e \Gamma_{1}}{\hbar}\left(N_{1}-N\right)
$$

and that of the right junction as:

$$
I_{R}=\frac{e \Gamma_{2}}{\hbar}\left(N-N_{2}\right)
$$

Steady state requires $I_{L}=I_{R}$ so that:

$$
\begin{aligned}
N & =2 \frac{\Gamma_{1} f\left(\epsilon, \mu_{1}\right)+\Gamma_{2} f\left(\epsilon, \mu_{2}\right)}{\Gamma_{1}+\Gamma_{2}} \\
I & =I_{L}=I_{R}=\frac{2 e}{\hbar} \frac{\Gamma_{1} \Gamma_{2}}{\Gamma_{1}+\Gamma_{2}}\left(f\left(\epsilon, \mu_{1}\right)-f\left(\epsilon, \mu_{2}\right)\right)
\end{aligned}
$$

Given the level $(\epsilon)$, broadening $\left(\Gamma_{1}, \Gamma_{2}\right)$ and the electrochemical potentials $\mu_{1}$ and $\mu_{2}$ of the two contacts, we can solve Eq. 19 for the current $I$. But we want to include charging effects in the calculations. Therefore, we add a potential $U_{S C}=\left\langle\delta v_{m o l}(r)\right\rangle$ due to the change in the number of electrons from the equilibrium value $2 f\left(\epsilon_{0}, E_{f}\right)$ :

$$
U_{S C}=U\left(N-2 f\left(\epsilon_{0}, E_{f}\right)\right)
$$

similar to a Hubbard model. We then let the level $\epsilon$ float up or down by this potential:

$$
\epsilon=\epsilon_{0}+U_{S C}
$$

Since the potential depends on the number of electrons, we need to calculate the potential using the self consistent procedure shown in Fig. 10.

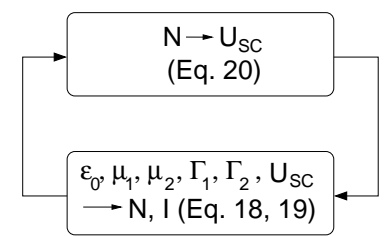

Figure 10: Illustration of the SCF procedure.

Note that, we should set $\eta=0.5$ (see Eq. 7) $\mu_{1}=E_{f}-0.5 \mathrm{eV}, \mu_{2}=$ $E_{f}+0.5 \mathrm{eV}$ corresponding to zero charge inside the molecule, since we are taking account of this charge separately by letting the molecular level $\epsilon$ float up or down, which could be viewed as a bias dependent adjustment of the effective $\eta$.

Once the converged solution is obtained, the current is calculated from Eq. 19. This very simple model captures much of the observed physics of molecular conduction. For example, the results obtained by setting $E_{f}=-5.0 \mathrm{eV}$, $\epsilon_{0}=-5.5 \mathrm{eV}, \Gamma_{1}=0.1 \mathrm{eV}, \Gamma_{2}=0.1 \mathrm{eV}$ are shown in Fig. 11 with $(U=1.0$ 

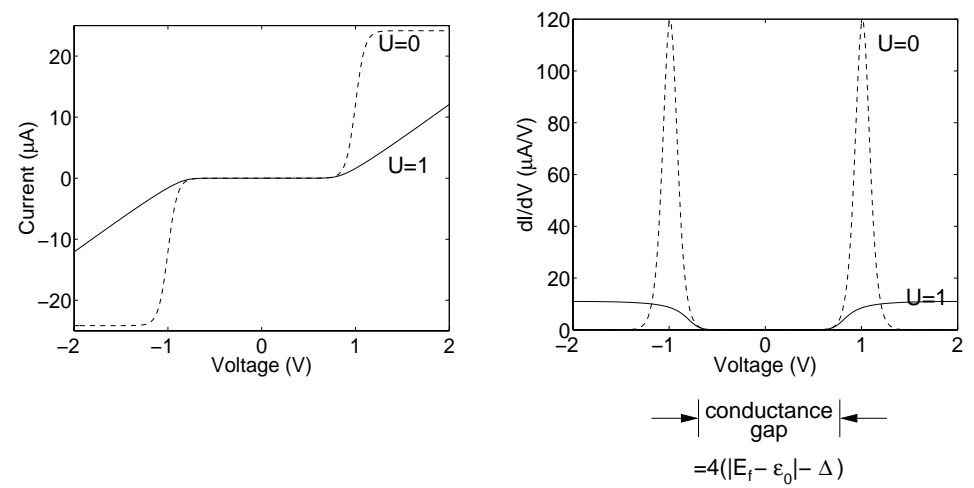

Figure 11: The current-voltage (I-V) characteristics (left) and conductancevoltage $(\mathrm{G}-\mathrm{V})$ (right) for our toy model with $E_{f}=-5.0 \mathrm{eV}, \epsilon_{0}=-5.5 \mathrm{eV}$ and $\Gamma_{1}=\Gamma_{2}=0.1 \mathrm{eV}$. Solid lines, charging effects included $(U=1.0 \mathrm{eV})$. Dashed line, no charging $(U=0)$. [37]

$\mathrm{eV})$ and without $(U=0 \mathrm{eV})$ charging effects. The finite width of the conductance peak (with $U=0$ ) is due to the temperature used in the calculations $\left(k_{B} T=0.025 \mathrm{eV}\right)$ since the levels are assumed to be discrete in this model. Note how the inclusion of charging tends to broaden the sharp peaks in conductance, even though we have not included any extra level broadening in this calculation. The size of the conductance gap is directly related to the energy difference between the molecular energy level and the Fermi energy. The current starts to increase when the voltage reaches $1 \mathrm{~V}$, which is exactly $2\left|E_{f}-\epsilon_{0}\right|$ as would be expected from $\eta$-theory (see Fig. $7(\mathrm{~b})$ ) with $\eta=0.5$. At higher voltages, when a chemical potential tries to cross the level, the level will be charged. The energy level thus shifts in energy (Eq. 21) if the charging energy is non-zero. Thus, for a small charging energy, the chemical potential easily crosses the level giving a sharp increase of the current. If the charging energy is large, the current increase gradually since the energy level follows the chemical potential due to the charging.

What determines the conductance gap? The above discussion shows that the conductance gap is equal to $4\left(\left|E_{f}-\epsilon_{0}\right|-\Delta\right.$ ) where $\Delta$ is equal to $\sim 4 k_{B} T$ (plus $\Gamma_{1}+\Gamma_{2}$ if broadening is included, see section 3.2), and $\epsilon_{0}$ is the HOMO or LUMO level whichever is closest to the Fermi energy, as pointed out in Ref. [38]. This is unappreciated by many who associate the conductance gap with the HOMOLUMO gap, which would be true if molecular levels remained fixed with respect to one contact as shown in Fig. 7(a). However, we believe that Fig. 7(b) is more appropriate at low current levels and what conductance measurements show is the gap between the Fermi energy and the nearest molecular level, which suggests that the same molecule can show different conductance gaps when contacted with different metals. Fig. 12 shows the I-V characteristics calculated 

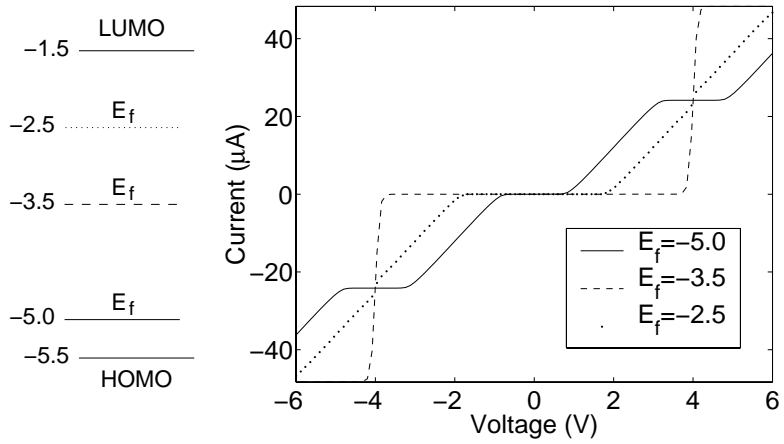

Figure 12: Right, the current-voltage (I-V) characteristics for the two level toy model for three different values of the Fermi energy $\left(E_{f}\right)$. Left, the two energy levels $(\mathrm{LUMO}=-1.5 \mathrm{eV}, \mathrm{HOMO}=-5.5 \mathrm{eV})$ and the three different Fermi energies $(-2.5,-3.5,-5.0)$ used in the calculations. (Other parameters used $\left.U=1.0 \mathrm{eV}, \Gamma_{1}=\Gamma_{2}=0.1 \mathrm{eV}\right)[37]$

using a two-level model (obtained by a straightforward extension of the onelevel model) with the Fermi energy located differently within the HOMO-LUMO gap giving different conductance gaps corresponding to the different values of $\left|E_{f}-\epsilon_{0}\right|$. Note that with the Fermi energy located halfway in between, the conductance gap is twice the HOMO-LUMO gap and the I-V shows no evidence of charging effects because the depletion of the HOMO is neutralized by the charging of the LUMO. This perfect compensation is unlikely in practice, since the two levels will not couple identically to the contacts as assumed in the model.

(a)

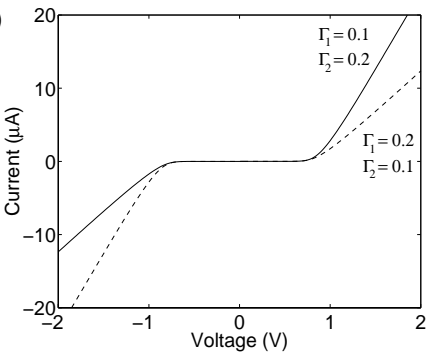

(b)

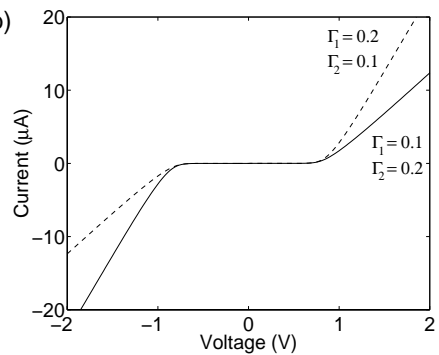

Figure 13: The current-voltage (I-V) characteristics for our toy model $\left(E_{f}=\right.$ $-5.0 \mathrm{eV}$ and $U=1.0 \mathrm{eV})$. (a) Conduction through HOMO $\left(E_{f}>\epsilon_{0}=-5.5\right.$ $\mathrm{eV})$. (b) Conduction through LUMO $\left(E_{f}<\epsilon_{0}=-4.5 \mathrm{eV}\right)$. Solid lines, $\Gamma_{1}=0.1$ $\mathrm{eV}<\Gamma_{2}=0.2 \mathrm{eV}$. Dashed lines, $\Gamma_{1}=0.2 \mathrm{eV}>\Gamma_{2}=0.1 \mathrm{eV}$. [37]

A very interesting effect that can be observed is the asymmetry of the I$\mathrm{V}$ characteristics if $\Gamma_{1} \neq \Gamma_{2}$ as shown in Fig. 13. This may explain several 
experimental results which show asymmetric I-V $[15,9]$ as discussed by Ghosh et al. [39]. Assuming that the current is conducted through the HOMO level $\left(E_{f}>\epsilon_{0}\right)$, the current is less when a positive voltage is applied to the strongly coupled contact, see Fig. 13(a). This is due to the effects of charging as we will discuss in more detail in section 5.2.3. Ghosh et al. also shows that this result will reverse if conduction is through the LUMO level. We can simulate this situation by setting $\epsilon_{0}$ equal to $-4.5 \mathrm{eV}, 0.5 \mathrm{eV}$ above the equilibrium Fermi energy $E_{f}$. The sense of asymmetry is now reversed as shown in Fig. 13(b). The current is larger when a positive voltage is applied to the strongly coupled contact. Comparing with STM measurements seems to favor the first case, i.e., conduction through the HOMO [39].

\subsection{Model with Broadening}

In Sec. 3.1, we treated the level $\epsilon$ as discrete, ignoring the broadening $\Gamma=\Gamma_{1}+\Gamma_{2}$ that accompanies the coupling to the contacts. To take this into account we need to replace the discrete level with a Lorentzian density of states $D(E)$ :

$$
D(E)=\frac{1}{2 \pi} \frac{\Gamma}{(E-\epsilon)^{2}+(\Gamma / 2)^{2}}
$$

and modifying Eqs. 18, 19 for $N$ and $I$ to include an integration over energy:

$$
\begin{aligned}
N & =2 \int_{-\infty}^{\infty} \mathrm{d} E D(E) \frac{\Gamma_{1} f\left(E, \mu_{1}\right)+\Gamma_{2} f\left(E, \mu_{2}\right)}{\Gamma_{1}+\Gamma_{2}} \\
I & =\frac{2 e}{\hbar} \int_{-\infty}^{\infty} \mathrm{d} E D(E) \frac{\Gamma_{1} \Gamma_{2}}{\Gamma_{1}+\Gamma_{2}}\left(f\left(E, \mu_{1}\right)-f\left(E, \mu_{2}\right)\right)
\end{aligned}
$$

The charging effect is included as before by letting the center $\epsilon$, of the molecular density of states, float up or down:

$$
\begin{aligned}
\epsilon & =\epsilon_{0}+U_{S C} \\
U_{S C} & =U\left(N-N_{0}\right)
\end{aligned}
$$

where the number of electrons at equilibrium is approximated by $N_{0}=2 f\left(\epsilon_{0}, E_{f}\right)$.

The results of calculating the I-V is presented in Fig. 14. Here, the same parameters as previously $\left(E_{f}=-5.0, \epsilon_{0}=-5.5, U=1\right.$ and $\Gamma_{1}=\Gamma_{2}=0.1$ $\mathrm{eV}$ ) were used. The only effect of level broadening is to smear out the I-V characteristic.

\subsection{Unrestricted Model}

In the previous examples (Figs. 11, 13) we have used values of $\Gamma_{1,2}$ that are smaller than the charging energy $U$. However, under these conditions one can expect single electron tunneling effects which are not captured by a "restricted 


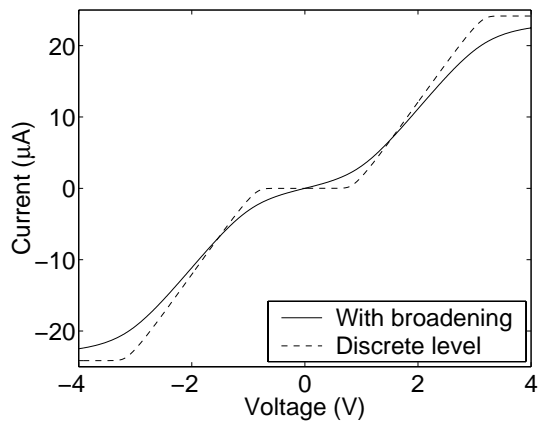

Figure 14: The current-voltage (I-V) characteristics: Solid line, include broadening of the level by the contacts. Dashed line, no broadening, same as solid line in Fig. 11. [37]

solution" which assumes that both spin orbitals see the same self-consistent field. However, an unrestricted solution, which allows the spin degeneracy to be lifted, will show these effects ${ }^{3}$. For example, if we replace Eq. 21 with:

$$
\begin{aligned}
\epsilon_{\uparrow} & =\epsilon_{0}+U\left(N_{\downarrow}-f_{0}\right) \\
\epsilon_{\downarrow} & =\epsilon_{0}+U\left(N_{\uparrow}-f_{0}\right)
\end{aligned}
$$

where the up-spin level feels a potential due to the down-spin electrons and vice-versa, then we obtain I-V curves as shown in Fig. 15.

If the SCF iteration is started with a spin degenerate solution, the same restricted solution as before is obtained. However, if the iteration is started with a spin non-degenerate solution a different looking I-V is obtained. The electrons only interact with the the electron of the opposite spin. Therefore, the chemical potential of one contact can cross one energy level of the molecule since the charging of that level only affects the opposite spin level. Thus, the I-V contains two separate steps separated by $U$ instead of a single step broadened by $U$.

For a molecule chemically bonded to a metallic surface, i.e., the PDT molecule bonded by the thiol group to a gold surface, the interaction $\Gamma$ is expected to be of the same magnitude or larger than $U$. Therefore, CB is not expected in this case [41]. However, if both interactions with the contacts are weak we should keep the possibility of $\mathrm{CB}$ and the importance of unrestricted solutions in mind.

\footnotetext{
${ }^{3}$ The unrestricted one-particle picture discussed here provides at least a reasonable qualitative picture of CB effects, though a complete description requires a more advanced many particle picture [40]. The one-particle picture leads to one of multiple possible states of the device depending on our initial guess, while a full many particle picture would include all states.
} 


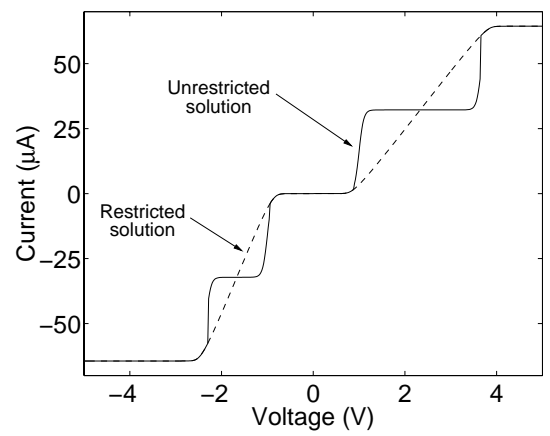

Figure 15: The current-voltage (I-V) characteristics for restricted (dashed line) and unrestricted solutions (solid line). $E_{f}=-5.0 \mathrm{eV}, \epsilon_{0}=-5.5 \mathrm{eV}, \Gamma_{1}=0.2$ $\mathrm{eV}>\Gamma_{2}=0.1 \mathrm{eV}$ and $U=1.0 \mathrm{eV}$. [37]

\section{A Realistic Multilevel Model}

The one-level toy model described in the last section includes the three basic factors that influence molecular conduction, namely, $E_{f}-\epsilon_{0}, \Gamma_{1,2}$ and $U$. However, real molecules typically have multiple levels that often broaden and overlap. Note that the two-level model (Fig. 12) in the last section treated the two levels as independent and such models can be used only if the levels do not overlap. In general we need a formalism that can do justice to multiple levels with arbitrary broadening and overlap. The non-equilibrium Green's function (NEGF) formalism described in this section does just that.

\subsection{Non-Equilibrium Green's Function (NEGF) Formal- ism}

In section 3.2 we obtained equations for the number of electrons, $N$ and the current, $I$ for a one-level model. It is useful to rewrite these equations in terms of the Green's function $G(E)$ which is defined as follows:

$$
G(E)=\left(E-\epsilon+i \frac{\Gamma_{1}+\Gamma_{2}}{2}\right)^{-1}
$$

The density of states $D(E)$ is proportional to the spectral function $A(E)$ defined as:

$$
\begin{aligned}
A(E) & =-2 \operatorname{Im}\{G(E)\} \\
D(E) & =\frac{A(E)}{2 \pi}
\end{aligned}
$$


while the number of electrons, $N$ and the current, $I$ can be written as:

$$
\begin{aligned}
N & =\frac{2}{2 \pi} \int_{-\infty}^{\infty} \mathrm{d} E\left(|G(E)|^{2} \Gamma_{1} f\left(E, \mu_{1}\right)+|G(E)|^{2} \Gamma_{2} f\left(E, \mu_{2}\right)\right) \\
I & =\frac{2 e}{h} \int_{-\infty}^{\infty} \mathrm{d} E \Gamma_{1} \Gamma_{2}|G(E)|^{2}\left(f\left(E, \mu_{1}\right)-f\left(E, \mu_{2}\right)\right)
\end{aligned}
$$

In the NEGF formalism the single energy level $\epsilon$ is replaced by a Hamiltonian matrix $[H]$ while the broadening $\Gamma_{1,2}$ is replaced by a complex energy-dependent self-energy matrix $\left[\Sigma_{1,2}(E)\right]$ so that the Green's function becomes a matrix given by:

$$
G(E)=\left(E S-H-\Sigma_{1}-\Sigma_{2}\right)^{-1}
$$

where $S$ is the identity matrix of the same size as the other matrices and the broadening matrices $\Gamma_{1,2}$ are defined as the imaginary (more correctly as the anti-Hermitian) parts of $\Sigma_{1,2}$ :

$$
\Gamma_{1,2}=i\left(\Sigma_{1,2}-\Sigma_{1,2}^{\dagger}\right)
$$

The spectral function is the anti-Hermitian part of the Green's function:

$$
A(E)=i\left(G(E)-G^{\dagger}(E)\right)
$$

from which the density of states $D(E)$ can be calculated by taking the trace:

$$
D(E)=\frac{\operatorname{Tr}(A S)}{2 \pi}
$$

The density matrix $[\rho]$ is given by, c.f., Eq. 32:

$$
\rho=\frac{1}{2 \pi} \int_{-\infty}^{\infty}\left[f\left(E, \mu_{1}\right) G \Gamma_{1} G^{\dagger}+f\left(E, \mu_{2}\right) G \Gamma_{2} G^{\dagger}\right] \mathrm{d} E
$$

from which the total number of electrons, $\mathrm{N}$ can be calculated by taking a trace:

$$
N=\operatorname{Tr}(\rho S)
$$

The current is given by, c.f., Eq. 33:

$$
I=\frac{2 e}{h} \int_{-\infty}^{\infty}\left[\operatorname{Tr}\left(\Gamma_{1} G \Gamma_{2} G^{\dagger}\right)\left(f\left(E, \mu_{1}\right)-f\left(E, \mu_{2}\right)\right)\right] \mathrm{d} E
$$

Equations 34 through 40 constitute the basic equations of the NEGF formalism which have to be solved self consistently with a suitable scheme to calculate the self-consistent potential matrix $\left[U_{S C}\right]$, c.f., Eq. 21 :

$$
H=H_{0}+U_{S C}
$$


where $H_{0}$ is the bare Hamiltonian (like $\epsilon_{0}$ in the toy model) and $U_{S C}$ is an appropriate functional of the density matrix $\rho$ :

$$
U_{S C}=F(\rho)
$$

This self-consistent procedure is essentially the same as in Fig. 10 for the one level toy model, except that scalar quantities have been replaced by matrices:

$$
\begin{aligned}
\epsilon_{0} & \rightarrow\left[H_{0}\right] \\
\Gamma & \rightarrow[\Gamma],[\Sigma] \\
N & \rightarrow[\rho] \\
U_{S C} & \rightarrow\left[U_{S C}\right]
\end{aligned}
$$

The sizes of all these matrices is $(n \times n), n$ being the number of basis functions used to describe the molecule. Even the self-energy matrices $\Sigma_{1,2}$ are of this size although they represent the effect of infinitely large contacts. In the remainder of this section, we will describe the procedure used to evaluate the Hamiltonian matrix $H$, the self-energy matrices $\Sigma_{1,2}$ and the functional " $F$ " used to evaluate the self-consistent potential $U_{S C}$ (see Eq. 42). But the point to note is that once we know how to evaluate these matrices, Eqs. 34 through 42 can be used straight forwardly to calculate the current.

Non-orthogonal basis: The matrices appearing above depend on the basis functions that we use. Many of the formulations in quantum chemistry use non-orthogonal basis functions and the matrix equations 34 through 42 are still valid as is, except that the elements of the matrix $[S]$ in Eq. 34 represents the overlap of the basis function $\phi_{m}(\bar{r})$ :

$$
S_{m n}=\int \mathrm{d}^{3} r \phi_{m}^{*}(\bar{r}) \phi_{n}(\bar{r})
$$

For orthogonal bases, $S_{m n}=\delta_{m n}$ so that $S$ is the identity matrix as stated earlier. The fact that the matrix equations 34 through 42 are valid even in a non-orthogonal representation is non self-evident and is discussed further in appendix A.

Incoherent Scattering: One last comment about the general formalism before we move onto the details of $H, \Sigma$ and $U_{S C}$. The formalism as described above neglects all incoherent scattering processes inside the molecule. In this form it is essentially equivalent to the Landauer formalism [42]. Indeed our expression for the current (Eq. 40) is exactly the same as in the transmission formalism with the transmission $T$ given by $\operatorname{Tr}\left(\Gamma_{1} G \Gamma_{2} G^{\dagger}\right)$. But it should be noted that, the real power of the NEGF formalism lies in its ability to provide a first principles description of incoherent scattering processes - something we do not address in this chapter and leave for future work. However, we do include an elementary model for scattering in order to take care of localized states that can otherwise cause numerical problems in the NEGF method. Since they 
are localized, they are not broadened and they do not contribute to the transmission. However, if they are occupied they contribute to the charge density matrix and are important to include in any SCF description. But their sharp structure makes it easy to miss their contribution while integrating over energy. One way to handle this problem is to integrate on a complex energy contour $[41,43,44]$. A more physical approach is to add a third contact which acts as a phase-breaking scatterer and broadens all states as first noted by Büttiker [3]. The non-equilibrium calculations in section 5 were performed with a probe described by the self energy $\Sigma_{p}=D_{p} \cdot S G(E) S$, where the constant $D_{p}=0.001$ $\mathrm{eV}^{2}$ and $G(E)$ the Green's function of the molecule. The Green's function:

$$
G=\left(E S-H-\Sigma_{1}-\Sigma_{2}-D_{p} \cdot S G S\right)^{-1}
$$

now has to be calculated self-consistently. A self-energy like this provides a broadening that is proportional to the density of states at every energy. This is physically more correct than the conventional "Büttiker" probe with a constant self-energy $\left(\Sigma_{p}=-i \eta S\right)$ and can be justified rigorously from the NEGF formalism for elastic (but incoherent) scattering.

Including this additional probe requires an additional term in the expression for the density matrix:

$$
\rho=\frac{1}{2 \pi} \int_{-\infty}^{\infty}\left[f\left(E, \mu_{1}\right) G \Gamma_{1} G^{\dagger}+f\left(E, \mu_{2}\right) G \Gamma_{2} G^{\dagger}+f\left(E, \mu_{p}\right) G \Gamma_{p} G^{\dagger}\right] \mathrm{d} E
$$

where $\mu_{p}$ is the electrochemical potential associated with this fictive probe. Strictly speaking this probe cannot be described by a single potential $\mu_{p}$ and requires the complete NEGF formalism for a proper treatment. However, since in our model this probe is merely intended to provide a small amount of broadening to avoid singularities due to localized states, we can treat it simply as a probe with a chemical potential given by:

$$
\mu_{p}=\frac{T_{1 p} \mu_{1}+T_{2 p} \mu_{2}}{T_{1 p}+T_{2 p}}
$$

where $T_{1 p}=\operatorname{Tr}\left(\Gamma_{1} G \Gamma_{p} G^{\dagger}\right)$ and $T_{2 p}=\operatorname{Tr}\left(\Gamma_{2} G \Gamma_{p} G^{\dagger}\right)$. It can be shown that this choice of $\mu_{p}$ ensures that the current $\left(I_{p}\right)$ into the probe is equal to zero [3]. In practice we can use any value of $\mu_{p}$ between $\mu_{1}$ and $\mu_{2}$ if the localized levels are well outside of this range. The current is given by:

$$
I=\frac{2 e}{\hbar} \int \mathrm{d} E T(E)\left(f\left(E, \mu_{1}\right)-f\left(E, \mu_{2}\right)\right)
$$

where $T(E)=T_{12}+\frac{T_{1 p} T_{2 p}}{T_{1 p}+T_{2 p}}, T_{12}=\operatorname{Tr}\left(\Gamma_{1} G \Gamma_{2} G^{\dagger}\right)$.

\subsection{Hamiltonian}

Now that we have outlined the basic equations of the NEGF formalism, let us talk about the details. The most important "detail" is to identify an appropriate 
Hamiltonian matrix to describe the molecule and the two contacts and then to evaluate the self-energy matrices $\Sigma_{1}$ and $\Sigma_{2}$ that account for the effects of the contacts on the molecule.

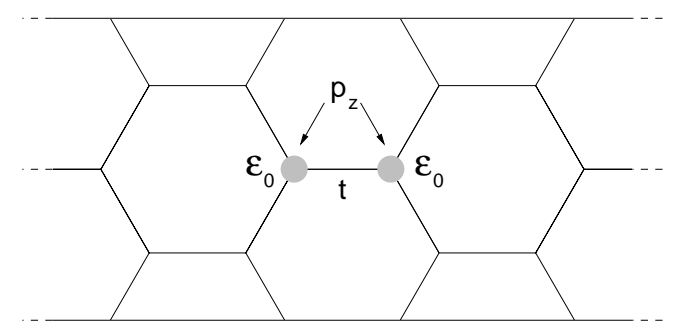

Figure 16: Two $p_{z}$ orbitals on a nanotube. Also shown are the onsite potential $\epsilon_{0}$ and hopping $t$.

The different approaches for obtaining the Hamiltonian matrix can broadly be divided into two categories: ab initio and semi-empirical. In ab-initio methods one chooses a set of basis functions (typically non-orthogonal) and evaluates the Hamiltonian matrix using a suitable scheme such as Hartree-Fock (HF) or density functional theory (DFT) to treat the electron-electron interactions. This requires the evaluation of "two-electron integrals" and a self-consistent iteration which make such methods time consuming. By contrast, semi-empirical approaches simply adjust the matrix elements based on a set of rules derived from a combination of theory and experimental observations. One example of a simple semi-empirical Hamiltonian is that commonly used to describe carbon nanotubes. The basis functions are chosen to be $p_{z}$-orbitals centered at each carbon atom (see Fig. 16) and the elements of the Hamiltonian matrix $H$ are assumed to be:

$$
\begin{array}{rll}
H_{i j} & =\epsilon_{0} \quad \text { if } j=i \\
& =-t \quad \text { if } j, i \text { are nearest neighbors } \\
& =0 \quad \text { otherwise }
\end{array}
$$

where $\epsilon_{0}, t$ are constants, typically 0 and $2.5 \mathrm{eV}$ respectively. The orbitals are assumed to be orthogonal, so that the overlap matrix $S$ is the identity matrix. Orthogonal parameterizations of this type based on $p_{z}$ orbitals are frequently used to describe molecules with $s p^{2}$ hybridization whose electronic properties are determined largely by the $p_{z}$-electrons. A more general method is the Extended Hückel Theory (EHT) which uses all valence orbitals of the atoms as the basis functions, e.g., carbon requires one $2 s$ and three $2 p$ orbitals as the basis. These atomic orbitals are approximated with Slater Type Orbitals [45] which allows the overlap matrix $S_{i j}=\langle i \mid j\rangle$ to be calculated efficiently. The matrix elements of the Hückel Hamiltonian $(H)$ are then described by the following equations:

$$
H_{i i}=\quad-V_{i}
$$




$$
H_{i j}=\frac{c}{2} S_{i j}\left(H_{i i}+H_{j j}\right) \quad i \neq j
$$

The diagonal elements of the Hamiltonian are approximated with the valence orbital ionization energies $\left(V_{i}\right)$ taken either from experimental data or calculated by more advanced methods. The off-diagonal elements are proportional to the overlap where the constant is usually taken as $c=1.75$. Thus, given a molecular geometry, the overlap matrix and thereafter the Hamiltonian can be calculated.
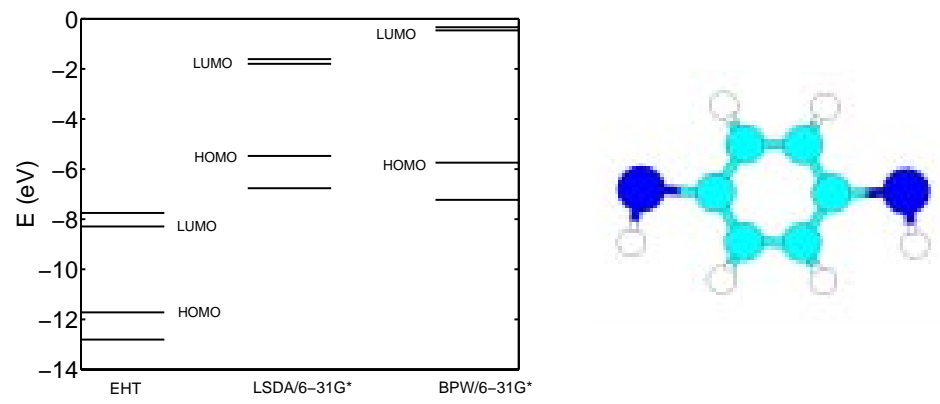

Figure 17: Energy levels (left) of the PDT molecule (right). EHT: Extended Hückel Theory. $L S D A / 6-31 \mathrm{G}^{*}$ : Density Functional Theory (DFT) with the $6-31 \mathrm{G}^{*}$ basis and the LSDA functional. $B P W / 6-31 \mathrm{G}^{*}$ : DFT with $6-31 \mathrm{G}^{*}$ basis and the Becke-Perdew-Wang (BPW) functional.

In this chapter we will present qualitative results based on the extended Hückel method which is widely used by chemists to describe the electronic structure of molecules and solids [46]. This approach requires only a fraction of the computational effort needed by the more elaborate ab initio methods and provides reasonably accurate quantitative results that give insight into the essential physics (or chemistry). Fig. 17 shows the energy levels of an isolated PDT molecule obtained from the EHT method compared with that obtained from Gaussian [47] using Density Functional Theory (DFT) with a $6-31 \mathrm{G}^{*}$ basis and the LSDA functional or the Becke-Perdew-Wang (BPW) functional for the self-consistent potential. Note that the EHT levels are 5-6 eV lower than the DFT levels but the relative energies are comparable and the wavefunctions of the HOMO and the LUMO levels are quite similar, see Fig. 18.

\subsection{Self Energy}

Once we have a Hamiltonian for the entire molecule-contact system, the next step is to "partition" the device from the contacts and obtain the self-energy matrices $\Sigma_{1,2}$ describing the effects of the contacts on the device. The contact will be assumed to be essentially unperturbed relative to the surface of a bulk metal, and this assumption is clearly not true for a few atoms near the moleculemetal interface. For this reason, the device should be defined so as to include 


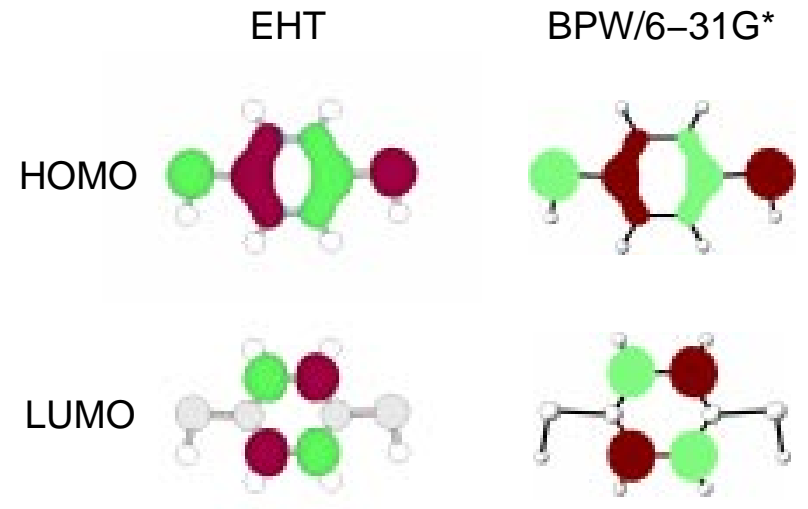

Figure 18: The wavefunctions of the HOMO (top) and LUMO (bottom) for EHT (left) and $B P W / 6-31 \mathrm{G}^{*}$ (right). LSDA also yields similar wavefunctions.

a cluster of metal atoms at each end which are affected by the presence of the molecule and the applied bias, see Fig. 19.

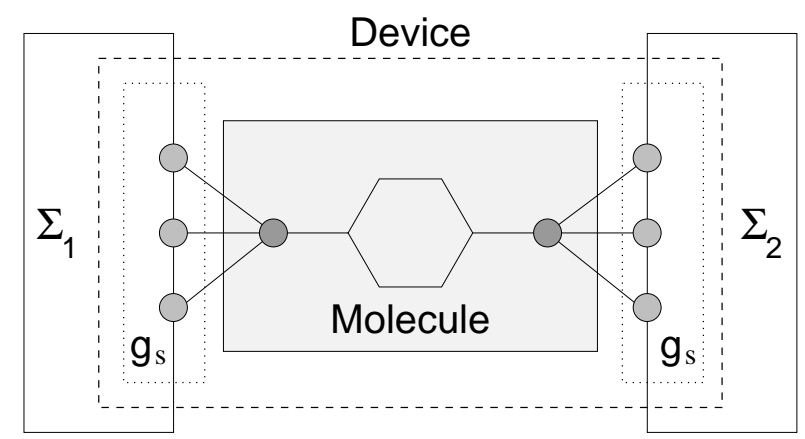

Figure 19: Molecule, device (including a few surface atoms), surface Green's function ( $g_{s}$ of the surface of the contacts) and Self-energies ( $\Sigma$ describing contacts without the surface metal atoms included in the device).

Once we have defined the "surface" atoms that we wish to include as part of our device, the next step is to compute the self-energy matrices $\Sigma_{1}$ and $\Sigma_{2}$ that describe the effect of the bulk contact (without the surface atoms that are included in the device). To do this we note that the Green's function for one of the isolated contacts (without the molecule) can be written as (the energy $E$ is assumed to have an infinitesimal imaginary part $i 0^{+}$):

$$
G=\left(\begin{array}{cc}
E S_{s}-H_{s} & E S_{s b}-H_{s b} \\
E S_{b s}-H_{b s} & E S_{b}-H_{b}
\end{array}\right)^{-1}=\left(\begin{array}{cc}
g_{s} & g_{s b} \\
g_{b s} & g_{b}
\end{array}\right)
$$


where $H_{s}$ is a finite sized matrix (describing the surface atoms included in the device) while $H_{b}$ is infinite (describing the rest of the contact). We wish to discover a $\Sigma$ of the same size as $H_{s}$ such that the surface Green's function $g_{s}$ is given by:

$$
g_{s}=\left(E S_{s}-H_{s}-\Sigma\right)^{-1}
$$

How do we discover the matrix $\Sigma$ ? The basic trick is that the surface Green's function $g_{s}$ can be computed exactly by making use of the periodicity of the infinite contact, using techniques that are standard in surface physics [32]. The details for an FCC (111) gold surface are described in appendix B. Once we have $g_{s}$ we can invert Eq. 56 to obtain $\Sigma$ :

$$
\Sigma=E S_{s}-H_{s}-g_{s}^{-1}
$$

This procedure can be used to obtain each of the matrices $\Sigma_{1}$ and $\Sigma_{2}$ assuming that they are unaffected by the presence of the molecule. Eq. 57 will provide a matrix of the same size as $H_{s}$ from which the full matrix (of the same size as the full device) is obtained by filling in with zeroes.

In some cases, it may be useful to define the molecule itself as the device without any surface atoms. In that case, we can obtain the self-energy $\Sigma$ by starting from the full Green's function:

$$
G_{T}=\left(\begin{array}{cc}
E S-H & E S_{d c}-H_{d c} \\
E S_{c d}-H_{c d} & E S_{c}-H_{c}
\end{array}\right)^{-1}=\left(\begin{array}{cc}
G & G_{d c} \\
G_{c d} & G_{c}
\end{array}\right)
$$

and partitioning it to discover a $\Sigma$ such that:

$$
G=(E S-H-\Sigma)^{-1}
$$

It is straightforward to show that:

$$
\Sigma=\left(E S_{d c}-H_{d c}\right)\left(E S_{c}-H_{c}\right)^{-1}\left(E S_{c d}-H_{c d}\right)=\tau g_{s} \tau^{\dagger}
$$

where $\tau$ is the non-zero part of $\left(E S_{d c}-H_{d c}\right)$ coupling the device to the surface Green's function corresponding to these surface atoms, which is determined following the procedure described in appendix B.

\subsection{Self-Consistent Potential}

Finally we need to identify the functional $F(\rho)$ that we will use to calculate the self-consistent potential $\left(U_{S C}\right)$, see Eq. 42. Ab initio techniques use methods based on HF or DFT to obtain these functionals which is numerically challenging and quantum chemistry programs like Gaussian incorporate many clever techniques to speed up these calculations which have been developed over the last thirty years. Although quantum chemistry software only address isolated molecules in equilibrium, see Fig. 20. Step 1 is really identical with what we need to do to address our problem. It is only step 2 that is different. We need to use the NEGF formalism to handle the non-equilibrium statistical mechanism of an open system. Since step 1 is identical it seems reasonable to start 
from a standard quantum chemistry software and modify step 2; this is exactly what has been done in Ref. [22], while others have set up the full self-consistent scheme [23].
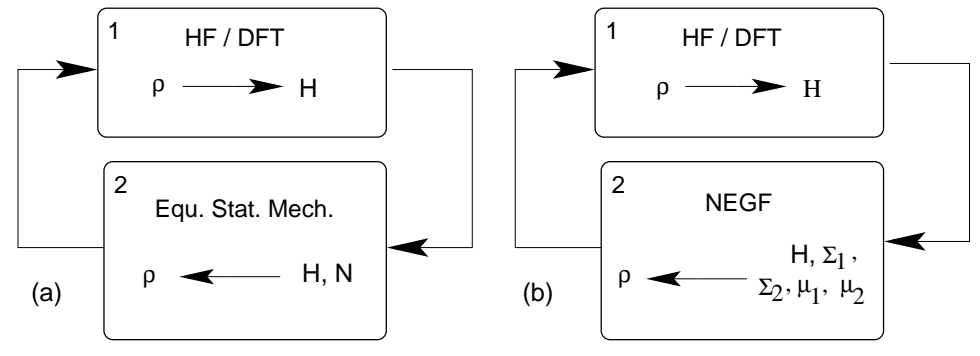

Figure 20: Comparison between (a) SCF quantum chemical methods and (b) the NEGF method.

Semi-empirical methods like the EHT use simpler functionals to impose selfconsistency. For example, the Pariser-Parr-Pople (PPP) method [48] is a tightbinding model designed to describe conjugated molecules. The $\pi$-electron system is described by one $p_{z}$ basis function per atom. The one-electron part of the Hamiltonian is similar to the EHT Hamiltonian, however, the basis is assumed to be orthogonal and the functional describing the electron-electron interactions is:

$$
H_{i j}^{e-e}=\delta_{i j}\left(\rho_{i i} \gamma_{i i}+\sum_{k(k \neq i)}\left(\rho_{k k}-Z_{k}\right) \gamma_{k i}\right)-\frac{1}{2} \rho_{i j} \gamma_{i j}
$$

where $\rho$ is the charge density matrix, $Z_{j}$ the charge of the atom without the $\pi$-electron and the one center two-electron integral $\gamma_{i j}$. The diagonal elements $\gamma_{i i}$ are obtained from experimental data and the off-diagonal elements $\left(\gamma_{i j}\right)$ are parameterized to describe a potential that decrease as the inverse of the distance $\left(1 / R_{i j}\right)$ :

$$
\gamma_{i j}=\frac{e^{2}}{4 \pi \epsilon_{0} R_{i j}+\frac{2 e^{2}}{\gamma_{i i}+\gamma_{j j}}}
$$

Calculations of the conductance through molecules using the PPP model has been carried out by Paulsson et al. [20]. However, this model has to be extended appropriately in order to be applied to non-orthogonal bases.

\section{Application of the NEGF Formalism}

We will now present some numerical results obtained using the Hückel Hamiltonian along with a computationally efficient toy model for the self-consistent potential $\left(U_{S C}\right)$ similar to that used in section 3. 
The potential of the molecule is approximated with a flat potential:

$$
U_{S C}=U_{a p p l}+F(\rho)=U_{a p p l}+U\left(N-N_{0}\right)
$$

where $U_{a p p l}=\left(U_{1}+U_{2}\right) / 2$ is the approximate solution to the Laplace equation (see Eq. 11), $U$ the charging energy and $N_{0}$ is the number of electrons in the neutral molecule. The advantage of a constant potential is that it merely shifts the molecular states up/down. Indeed we can treat the molecular states as unchanged and simply adjust the chemical potentials of the contacts by $U_{S C}$ :

$$
\mu_{1,2}=E_{f}+U_{1,2}-U_{S C}
$$

where $U_{1,2}$ is the applied potential. The chemical potential of the probe was set to $\mu_{p}=\left(\mu_{1}+\mu_{2}\right) / 2$ as discussed following Eq. 50. We can then calculate the number of electrons on the device by summing over the contacts and the probe:

$$
N=N_{1}\left(\mu_{1}\right)+N_{2}\left(\mu_{2}\right)+N_{p}\left(\mu_{p}\right)
$$

The functions $N_{1,2, p}$ (Eq. 49):

$$
N_{1,2, p}(\mu)=\frac{1}{2 \pi} \int_{-\infty}^{\infty} \operatorname{Tr}\left(G \Gamma_{1,2, p} G^{\dagger}\right) f(E, \mu) \mathrm{d} E
$$

can be calculated once ${ }^{4}$, stored, and then reused in every SCF step for each applied potential. Equations 63-66 are then solved self-consistently. Since the integrals only have to be calculated once, the SCF calculations are fast. The current is then found from Eq. 51.

This approach captures the basic physics of charging while being computationally inexpensive. However, the assumption of a constant potential across the device is hard to justify if we include a large cluster of metal atoms at each end of the device which will tend to have the same potential as the bulk contact. For this reason, the results we present here have been obtained using a device that consists of just the molecule without any additional metal atoms, using Eq. 60 from section 4.3 to calculate the self-energy.

\subsection{Gold Contacts}

Gold (111) films are commonly used to form the contacts to molecules. In Fig. 21, we show the surface density of states (DOS) for a gold (111) surface calculated from the surface Green's function taking the FCC lattice of bulk gold (nearest neighbor (n.n.) distance $2.885 \AA$ ) into account. In the Hückel model each gold atom is described by three $5 p$-, five $5 d$ - and one $6 s$-orbital. Each of these orbitals contributes to the total density of states as shown. The features

\footnotetext{
${ }^{4}$ Changing the potential of the contacts shifts the energy bands in the contacts. This shift gives a change in $G$ and $\Gamma_{1,2}$ in Eq. 66 . However, this shift can be ignored for applied voltages much less than the bandwidth of the contacts.
} 
of the d- and p-band frame a region of nearly constant density of states from the s-band. The Fermi energy of gold, calculated in the Hückel model using a $51 \times 51 \times 51$ cluster with periodic boundary conditions, is $E_{f}=-9.50 \mathrm{eV}$. Notice that this value of $E_{f}$ differs from the (negative) workfunction for gold ( $\sim-5.3$ $\mathrm{eV}$ ) because the Hückel energy levels are $4-5 \mathrm{eV}$ lower than the correct values relative to vacuum (see Fig. 17). At the Fermi energy the s-band dominates the DOS and any related properties such as the broadening of the molecular levels.
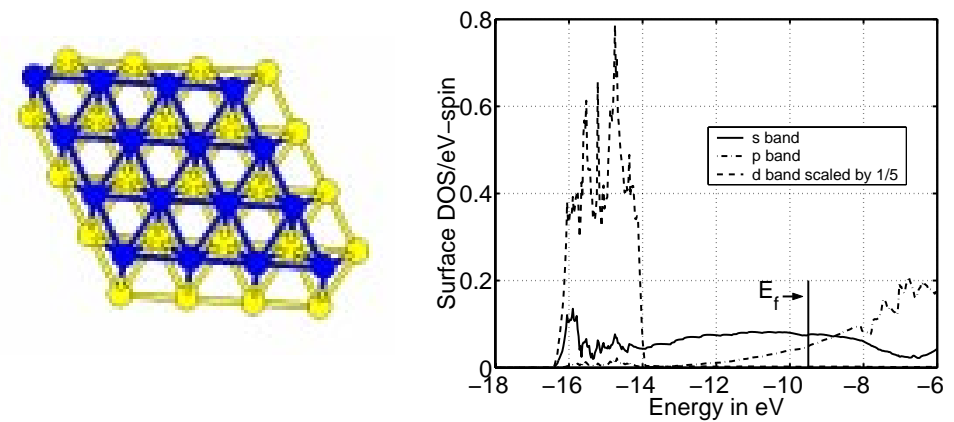

Figure 21: Left, two layers (with different shadings) of the $\mathrm{Au}$ (111) surface. Right, contributions to the surface density of states from the $5 p, 5 d$ and $6 s$ orbitals.

The conductance through a Quantum Point Contact (QPC), i.e., a thin (a few gold atoms thick) gold wire (see Fig. 1), has been shown experimentally [49] to equal the number of modes (roughly equal to the number of gold atoms in the cross section), times the quantum conductance $\frac{e^{2}}{\pi \hbar} \sim 77.5 \mu \mathrm{A} / \mathrm{V} \sim(12.9 \mathrm{k} \Omega)^{-1}$. Therefore, a good test of our method is to calculate the I-V of a linear chain of six gold atoms sandwiched between two gold (111) contacts. The distances between the gold atoms in the chain was set equal to the n.n. distance in bulk gold ( 2.885 $\AA$ ) although the actual spacing in experiments is somewhat controversial. The calculated I-V compares well with the linear I-V having a slope of $\frac{e^{2}}{\pi \hbar}$ shown in Fig. 1.

\subsection{Phenyl Dithiol}

Now that we have a model for a QPC, it is straightforward to replace the $A u_{6}$ molecule with any other molecule provided we know how it is bonded to the gold surface. For phenyl dithiol (PDT) it is known that [50] the sulfur atoms bond to the surface on the center of a gold triangle with a gold-sulfur bond length of $2.53 \AA$ (Fig. 22). 

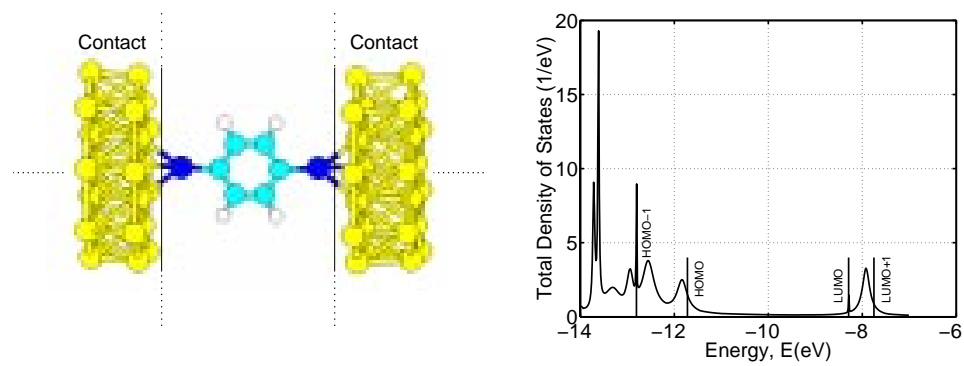

Figure 22: Left, the PDT molecule chemically bound to the Au (111) surface by the sulfur atoms. Right, total DOS on the PDT molecule vs. Energy. Also shown are the discrete energy levels of an isolated PDT molecule.

\subsubsection{Equilibrium Properties}

Connecting the molecule to the contacts broadens and shifts the discrete states of the molecule as shown in Fig. 22. The broadening of the DOS depends on the strength of the coupling to the contacts as well as the wavefunction of the particular state. This is illustrated by the large broadening of the HOMO while the LUMO is a sharp spike (almost invisible in the figure) since the LUMO is localized to the benzene ring.

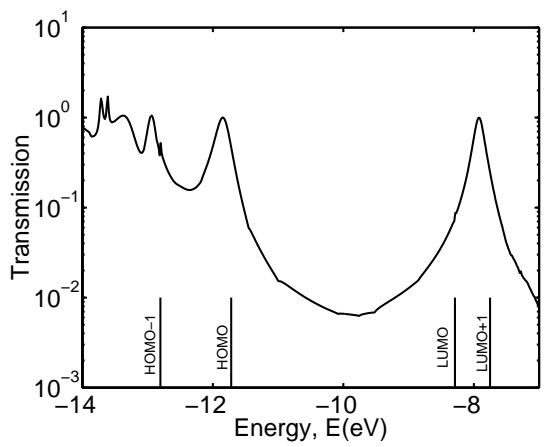

Figure 23: Transmission through the PDT molecule attached to gold (111) contacts. The discrete energy levels of an isolated PDT molecule are also shown.

The transmission through a PDT molecule often resembles the DOS closely although this need not always be true. If the wavefunction (for one level) is asymmetric it may couple strongly to only one contact giving a wide DOS peak but a narrow transmission peak. However, for the PDT molecule this is evidently not the case (see Fig. 23) and the transmission closely resembles the DOS (note that one is plotted on a linear scale while the other is plotted on a logarithmic scale). Note also that, despite the strong chemical bond between 
the sulfur and gold DOS and transmission are peaked around the states of the isolated molecule. This shows the localized character of the chemical bond, i.e., the sulfur atom partially insulates the $\pi$-electron states of the molecule. By contrast, the transmission for a QPC is nearly constant as evidenced by the linear I-V.

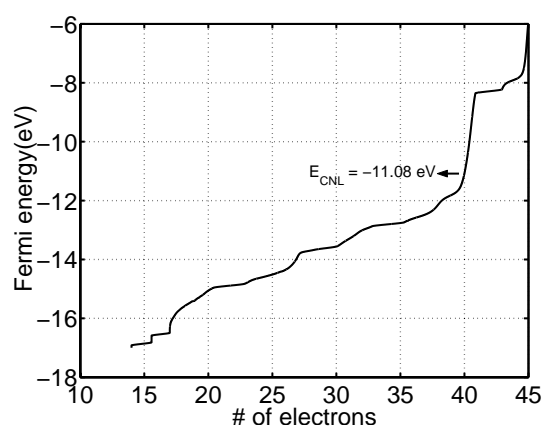

Figure 24: Equilibrium chemical potential as a function of number of electrons on the molecule. Note the large change in the Fermi energy in response to small changes in the number of electrons from 40; this makes the correct location of the Fermi energy extremely sensitive to experimental uncertainties as well as numerical errors.

Where is the Fermi energy? Connecting the molecule to the contact will in general result in charge transfer between the contacts and molecule (see section 2.1 and Fig. 5). The direction and magnitude of the charge transfer depend on the chemical potentials of the isolated parts together with the charging energy of the molecule. The chemical potential for the contacts is calculated to be $E_{f}=$ $-9.50 \mathrm{eV}$, as discussed in section 5.1. To locate the charge neutrality level (CNL) of the molecule, the chemical potential is plotted against the number of electrons in the molecule (Fig. 24). For the PDT molecule (without two hydrogens) the molecule is neutral with 40 electrons giving a CNL of $E_{N}^{0}=-11.08 \mathrm{eV}$, which is in the HOMO-LUMO gap and closer to the HOMO level than the LUMO of the molecule. Referring to Fig. 24 and the related discussion, it can be seen that there are two limiting possibilities: (1) If $U D \ll 1$, then the molecular levels remain unchanged and $E_{f}=-9.5 \mathrm{eV}$ is closer to the LUMO (see Fig. 22) and (2) if $U D \gg 1$, then the molecular levels float up to align $E_{f}$ with $E_{N}^{0}$. In the second case, we could view the molecular levels as fixed while $E_{f}$ floats down to $E_{N}^{0}=-11.08 \mathrm{eV}$, thereby putting $E_{f}$ very close to the HOMO. The "truth" we believe is closer to the second case but we leave it to future work to settle the question unambiguously. In the following examples, we have used values of $E_{f}$ lying between $-10.5 \mathrm{eV}$ and $-11.0 \mathrm{eV}$ as indicated. 


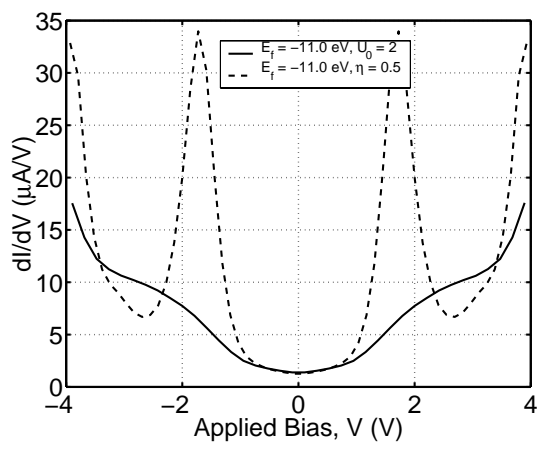

Figure 25: Conductance vs. Voltage (G-V) for PDT (symmetric coupling). Solid line, SCF solution with $U=2 \mathrm{eV}$. Dashed line, $U=0$ for comparison.

\subsubsection{Non Equilibrium Properties}

The I-V characteristics for the PDT molecule is shown in Fig. 25 for both $U=2 \mathrm{eV}$ and $U=0$. With $U=0$, the conductance corresponds to resonant tunneling through molecular states that are fixed. This gives rise to peaks in the conductance at the energy of the molecular energies. Broadening by the contacts cause the width of the peaks, a small broadening by the finite temperature $\left(k_{B} T=0.025 \mathrm{eV}\right)$ used in the calculations is also present. In Fig. 25 (dashed line, $U=0$ ), the peak is positioned at $V_{a p p l} \sim 1.5 \mathrm{~V}$ which corresponds to $2\left(E_{f}-E_{H O M O}\right)$. The experimental results on PDT do not show pronounced peaks. Therefore, a fit to the experimental data, with $U=0$, is only possible if a significant amount of inelastic scattering is included.

With $U=2 \mathrm{eV}$, however, the peaks broaden, even without any additional scattering, due to charging. When the applied voltage makes the chemical potential of one contact cross the HOMO level, the molecule loses a fraction of an electron making it positively charged. This makes the molecular energy levels float down and partially follow the chemical potential in the contact. Therefore, the pronounced peak in $U=0$ theory is replaced by a slower increase in the $\mathrm{I}-\mathrm{V}$ giving a plateau in the G-V. As the voltage is increased further, both the HOMO and LUMO level are contributing to the transmission. Here, the molecule lose electrons from the HOMO level but the other contact inject electrons in the LUMO. Therefore, the chemical potentials can cross the energy levels of the molecule without extensive charging. This gives rise to the large increase in the $\mathrm{G}-\mathrm{V}$ at high voltages $\left(V_{a p p l}>3.5 \mathrm{~V}\right)$.

The energy difference between the Fermi energy and the energy levels of the molecule determine the size of the conductance gap. In Fig. 26, the G-V for two different values of $E_{f}$ is shown. The dashed lines shows the results of moving the Fermi energy, compared with the solid line, $0.25 \mathrm{eV}$ further away from the HOMO level. The conductance gap increases as we would expect. The increase is a little less than the expected $0.5 \mathrm{~V}$ due to the change in charging of the 


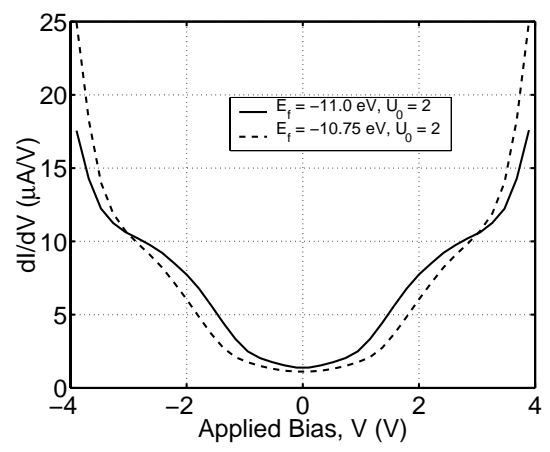

Figure 26: G-V for PDT (symmetric coupling) for two different $E_{f}$.

molecule at equilibrium. However, the second peak, which is largely unchanged, in the G-V starts at a slightly lower applied voltage since the Fermi energy of the contacts is closer to the LUMO.

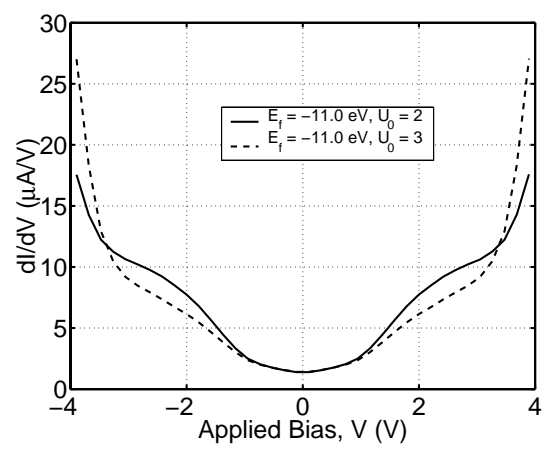

Figure 27: G-V for PDT (symmetric coupling) for two different charging energies $(U)$.

The second parameter in the SCF calculations is the charging energy of the molecule which is estimated to be $U=2.0 \mathrm{eV}$ from a Pariser-Parr-Pople Hamiltonian [20]. Increasing the charging energy from $U=2.0 \mathrm{eV}$ to $U=3.0$ $\mathrm{eV}$ drags out the G-V plot (Fig. 27) due to a slower crossing of the HOMO level by the chemical potential. However, the second peak in the $\mathrm{G}-\mathrm{V}$ is not changed, since it corresponds to emptying the HOMO while filling the LUMO-level.

\subsubsection{Asymmetric Coupling to the Contacts}

In most experimental measurements there is some doubts about the quality of the chemical bond between the molecule and contact. Even if there is a well 
formed self assembled monolayer on one of the metallic contacts, the bonding with the other contact is probably not perfect since it is simply evaporated. To model a device where one side of the molecule is strongly coupled to the contact while the other side is weakly coupled we performed calculations on a system where we artificially reduce the self-energy of one contact by a constant factor $C^{2}$. This is the same as multiplying the interaction elements $(\tau)$ of the Hamiltonian by $C$, i.e., decreasing the coupling between the device and contact by $C$.

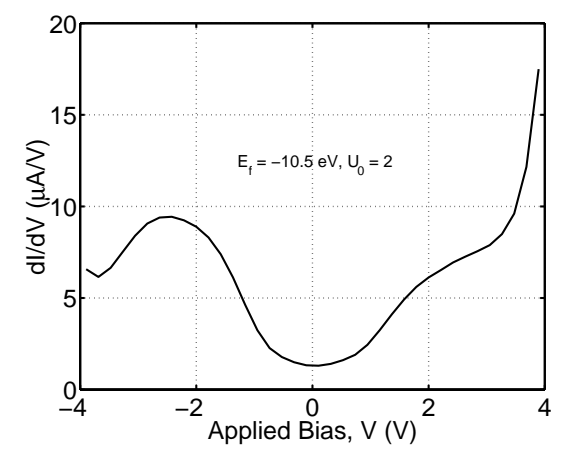

Figure 28: G-V for PDT (asymmetric coupling).

In Fig. 28, the G-V of the PDT molecule with a weaker coupling to the right contact is shown. Here we used $C^{2}=0.6$ corresponding to a reduction of the molecule contact overlap $\tau$ by $\sim 0.77$. Even this relatively weak reduction ${ }^{5}$ of the coupling leads to marked changes in the G-V. For negative $V_{a p p l}\left(\mu_{1}>\mu_{2}\right)$, the chemical potential of the weakly coupled contact $\left(\mu_{2}\right)$ can cross the HOMO level of the molecule without extensive charging giving a peak in the G-V that is only slightly dragged out. But for positive bias, where the chemical potential of the strongly coupled contact $\left(\mu_{1}\right)$ crosses the HOMO level, the effects of charging drags out the G-V peak. The resulting asymmetric I-V seems to explain the experimental observation in Fig. 10 of Ref. [15] as noted by Ghosh et al. [39].

\section{Summary and Conclusions}

We started with an intuitive description of the current-voltage (I-V) characteristics of molecules (section 2) and then introduced several simple toy models that captures the basic physics (section 3). These toy models were also used to motivate the rigorous Non-Equilibrium Green's Function (NEGF) theory (section 4) and applied using the Hückel Hamiltonian to a Phenyl Dithiol molecule coupled to gold contacts (section 5).

\footnotetext{
${ }^{5} \mathrm{~A}$ change of 0.77 in the coupling matrix $\tau$ corresponds to an estimated elongation of the $\mathrm{S}$ - $\mathrm{Au}$ bond of the order of $\sim 0.15 \AA$.
} 
With both the simple toy models and the NEGF-Hückel calculations we find that the three most important properties that determine the current-voltage (I-V) characteristics of a molecule are:

1. The position of the energy levels of the molecule relative to the Fermi energy of the contacts. Using simple toy models the conductance gap is seen to correspond to $4\left|E_{f}-\epsilon_{0}\right|$, i.e., the difference of the Fermi energy and the closest molecular energy level. We believe that more theoretical and experimental effort should be focused on establishing the precise location of $E_{f}$ for different metal-molecule "hetrostructures".

2. The broadening of the energy levels due to the coupling to the contacts determine the magnitude of the current through the molecule.

3. The charging of the molecule broadens the conductance peaks by the charging energy per electron $U$. This can explain many experimental observations without invoking any additional inelastic scattering. Also, the asymmetry of this charging effect can explain why symmetric molecules give asymmetric I-V when coupled unequally to the two contacts.

Although most observed I-V characteristics have the shape shown in Fig. 2 and can be understood in terms of the three parameters listed above, more interesting I-V can be expected to arise with cleverly engineered molecular structures. The NEGF formalism coupled with the Hückel Hamiltonian as described in section 4 should be useful in the theoretical investigation of such effects and a simple implementation of this model (which is an improved version of the one installed earlier) can be accessed by readers through the Purdue Simulation Hub ("www.nanohub.purdue.edu").

\section{Acknowledgments}

Sections 2, 3 and 4 are based on material from a forthcoming book by one of the authors (S.D.) entitled Quantum Phenomena: From Atoms to Transistors. It is a pleasure to acknowledge helpful feedback from Mark Ratner, Phil Bagwell and Mark Lundstrom. The authors are grateful to Prashant Damle and Avik Ghosh for helpful discussions regarding the ab initio models and to Manoj Samanta and Weidong Tian for their contributions to the older version of the semi-empirical Hückel based model. This work was supported by the NSF/ARO under Grants No. 9708107 - DMR / No. 0085516 - EEC and the DARPA/ARO under Grant No. DAAH04-96-1-0437.

\section{A NEGF in Non-Orthogonal Basis}

This appendix discusses the conceptual issues related to the use of a nonorthogonal representation in an NEGF calculation (Eqs. 34-40). 
Non-orthogonal bases are commonly used in quantum chemistry [51] and we could use the standard approach to transform $H, \Sigma$ into an orthogonal representation $\widetilde{H}, \widetilde{\Sigma}[51]$ :

$$
\begin{aligned}
\widetilde{H} & =S^{-1 / 2} H S^{-1 / 2} \\
\widetilde{\Sigma} & =S^{-1 / 2} \Sigma S^{-1 / 2}
\end{aligned}
$$

and define a transformed Green's function:

$$
\widetilde{G}=(E I-\widetilde{H}-\widetilde{\Sigma})^{-1}
$$

where I is the identity matrix which could be viewed as $\widetilde{S}$, the orthogonalized version of $S$. It is easy to show that:

$$
\begin{aligned}
G & =S^{-1 / 2} \widetilde{G} S^{-1 / 2} \\
\Gamma & =S^{1 / 2} \widetilde{\Gamma} S^{1 / 2} \\
G \Gamma_{1,2} G^{\dagger} & =S^{-1 / 2}\left[\widetilde{G} \widetilde{\Gamma}_{1,2} \widetilde{G}^{\dagger}\right] S^{-1 / 2} \\
\Gamma_{1} G \Gamma_{2} G^{\dagger} & =S^{1 / 2}\left[\widetilde{\Gamma}_{1} \widetilde{G} \widetilde{\Gamma}_{2} \widetilde{G}^{\dagger}\right] S^{-1 / 2} \\
\operatorname{Tr}\left(\Gamma_{1} G \Gamma_{2} G^{\dagger}\right) & =\operatorname{Tr}\left(\widetilde{\Gamma}_{1} \widetilde{G} \widetilde{\Gamma}_{2} \widetilde{G}^{\dagger}\right)
\end{aligned}
$$

One could argue that if Eqs. 34-40 are valid in the orthogonal representation (denoted by the tilde ), then Eqs. 67-74 can be used to show that they will be will be valid for the non-orthogonal representation as well. Note that:

$$
\begin{gathered}
\widetilde{A}=S^{1 / 2} A S^{1 / 2} \quad \Rightarrow \quad 2 \pi D(E)=\operatorname{Tr}(\widetilde{A})=\operatorname{Tr}(A S) \\
\widetilde{\rho}=S^{1 / 2} \rho S^{1 / 2} \quad \Rightarrow \quad N=\operatorname{Tr}(\widetilde{\rho})=\operatorname{Tr}(\rho S)
\end{gathered}
$$

This shows that when calculating the number of electrons, $N$ or the density of states $D(E)$, we should multiply $\rho$ or $A$ by $S$ before taking the trace as we did in Eqs. 37 and 39. Note that quantities like $H, \Sigma, \Gamma$ transform differently from quantities like $G, A, \rho$. Their transformation rules are similar to covariant and contravariant tensors respectively [52].

It is important to note that we are obtaining $\Sigma$ by partitioning in a nonorthogonal representation and then transforming it to yield $\widetilde{\Sigma}$ (see Eq. 68). The result is NOT the same as partitioning in the orthogonal representation: Not unless the overlap matrix is block diagonal, such that $S_{d c}=S_{c d}=[0]$. This can be shown mathematically but the physical reason is obvious. If there is any overlap between the device and the contact then the process of orthogonalization will mix the two spaces. The device subspace $\widetilde{d}$ in the orthogonal representation will not be the same as the device subspace $d$ in the non-orthogonal representation, but will include some of the contact subspace $c$ as well. In that case, partitioning in the orthogonal representation and in the non-orthogonal representation correspond to using two different physical "partitions" and there is no reason 
to expect any equivalence. A non-zero $S_{d c}$ introduces an element of ambiguity into the process of partitioning, not unlike the ambiguity associated with the different schemes for charge partitioning within a molecule (e.g. Mulliken and Löwdin population analysis [51]).

\section{B Surface Green's Function}

This appendix discusses the procedure for calculating the surface Green's function of a given contact used to evaluate the self-energy from Eq. 60 [53].

Consider a semi-infinite solid whose overall Green's function can be written in the form:

$$
G=(E S-H)^{-1}=\left(\begin{array}{cccc}
\alpha & \beta & 0 & \cdots \\
\beta^{\dagger} & \alpha & \beta & \\
0 & \beta^{\dagger} & \alpha & \\
\vdots & & & \ddots
\end{array}\right)^{-1}
$$

where $\alpha$ is the matrix $E S-H$ corresponding to one isolated layer of the solid and the matrix $\beta$ provides the inter-layer coupling, see Fig. 29. Each of these

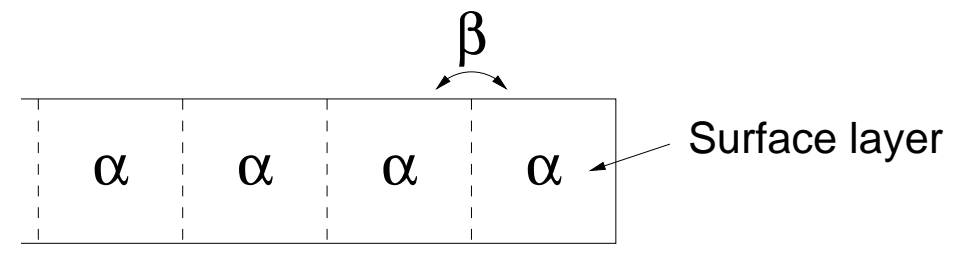

Figure 29: The division of the semi-infinite contact into layers.

matrices are infinitely large since each layer is infinite in the two dimensions parallel to the layers. We can simplify the problem by a two dimensional unitary transformation to k-space, i.e., Fourier transform the two dimensions parallel to the layers [54]:

$$
\begin{aligned}
& \alpha(\bar{k})=\alpha_{\bar{k}, \bar{k}}=\sum_{i} \alpha_{i j} e^{-i \bar{k} \cdot\left(\bar{r}_{i}-\bar{r}_{j}\right)} \\
& \beta(\bar{k})=\beta_{\bar{k}, \bar{k}}=\sum_{i} \beta_{i j} e^{-i \bar{k} \cdot\left(\bar{r}_{i}-\bar{r}_{j}\right)}
\end{aligned}
$$

where $\alpha_{i j}$ correspond to the matrix elements of the layer subspace corresponding to atom $i, j$, i.e., the interaction atom $j$ to $i$, and similar for $\beta_{i j}$. Note that, $\alpha(\bar{k})$ and $\beta(\bar{k})$ are matrices with dimension equal to the number of orbitals in the unit cell. The periodicity of each layer ensures that the sum is independent of the value of ' $j$ ' and that the different $\bar{k}$ values are completely decoupled so 
that we can calculate the surface Green's function independently for each $\bar{k}$ :

$$
G(\bar{k})=\left(\begin{array}{cccc}
\alpha(\bar{k}) & \beta(\bar{k}) & 0 & \cdots \\
\beta^{\dagger}(\bar{k}) & \alpha(\bar{k}) & \beta(\bar{k}) & \\
0 & \beta^{\dagger}(\bar{k}) & \alpha(\bar{k}) & \\
\vdots & & & \ddots
\end{array}\right)^{-1}=\left(\begin{array}{cc}
g_{s}(\bar{k}) & \cdots \\
\vdots & \ddots \\
&
\end{array}\right)
$$

where we are only interested in the surface Green's function $g_{s}(\bar{k})$ which can be shown to satisfy the recursive relation:

$$
g_{s}(\bar{k})=\left(\alpha(\bar{k})-\beta(\bar{k}) g_{s}(\bar{k}) \beta^{\dagger}(\bar{k})\right)^{-1}
$$

This equation can either be solved analytically (if the matrices $\alpha$ and $\beta$ are one-dimensional) or by iteration starting from a reasonable guess for $g_{s}(\bar{k})$. We can then transform it back to real space by summing over the allowed $\bar{k}$ vectors (using periodic boundary conditions with $N$ unit cells in each direction):

$$
g_{s}\left(\bar{r}_{i}-\bar{r}_{j}\right)=\frac{1}{N^{2}} \sum_{\bar{k}} g_{s}(\bar{k}) e^{i \bar{k} \cdot\left(\bar{r}_{i}-\bar{r}_{j}\right)}
$$

Now we turn to the details of calculating the surface Green's function for a Gold (111) surface. To find the $\alpha(\bar{k})=E S(\bar{k})-H(\bar{k})$ and $\beta(\bar{k})$ we need the overlap and Hamiltonian matrices in real space. These were obtained from the Hückel Hamiltonian and overlap matrix of a 13 atom gold cluster, see Fig. 30. From the matrices of the cluster, the sub-matrices $S_{i 1}$ and $H_{i 1}$ were obtained, where we use $j=1$ since the sums in Eq. 78 and 79 are independent of $j$.
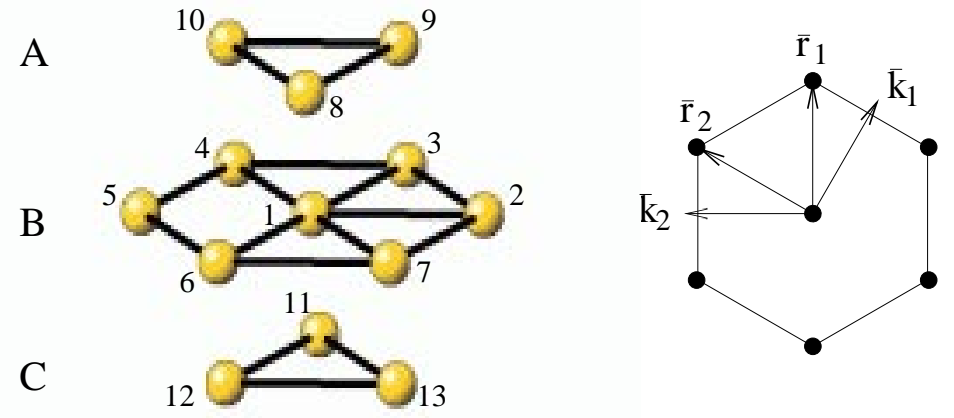

Figure 30: Left, numbering of gold atoms in the 13 atom Au cluster. Note that the plane formed by atoms 1-7 is the FCC (111) surface. Right, basis vectors and reciprocal basis used in the two dimensional Fourier transform.

Transforming the two real space dimensions (forming the plane of Au atoms 1-7 in Fig. 30) into k-space (assuming only nearest neighbor coupling) gives, 
c.f., Eqs. 78 and 79:

$$
\begin{aligned}
& \alpha(\bar{k})=\sum_{i=1}^{7} e^{-i \bar{k} \cdot\left(\bar{r}_{i}-\bar{r}_{1}\right)}\left(E S_{i 1}-H_{i 1}\right) \\
& \beta(\bar{k})=\sum_{i=8}^{10} e^{-i \bar{k} \cdot\left(\bar{r}_{i}-\bar{r}_{1}\right)}\left(E S_{i 1}-H_{i 1}\right)
\end{aligned}
$$

One way to simplify these calculations is to note that the product $\bar{k} \cdot \bar{r}$ always can be written:

$$
\bar{k} \cdot \bar{r}=\left(k_{1} \bar{k}_{1}+k_{2} \bar{k}_{2}\right) \cdot\left(r_{1} \bar{r}_{1}+r_{2} \bar{k}_{2}\right)=k_{1} r_{1}+k_{2} r_{2}
$$

from the definition of the resiprocal lattice vectors $\bar{k}_{1}$ and $\bar{k}_{2}$ (see Fig. 30). Using periodic boundary conditions ( $N$ unit cells in directions $\bar{k}_{1}$ and $\left.\bar{k}_{2}\right)$ we see that $k_{1}=\frac{2 \pi m}{N}$ and $k_{2}=\frac{2 \pi n}{N}$ where $m$ and $n$ are integers. This makes the transformation back to real space painless:

$$
g_{s}\left(\bar{r}_{i}-\bar{r}_{j}\right)=\frac{1}{N^{2}} \sum_{m, n=0}^{N-1} e^{i \bar{k} \cdot\left(\bar{r}_{i}-\bar{r}_{j}\right)} g_{s}(\bar{k})
$$

where $\bar{k}=\frac{2 \pi m}{N} \bar{k}_{1}+\frac{2 \pi n}{N} \bar{k}_{2}$. 


\section{References}

[1] P. Avouris, P. G. Collins, and M. S. Arnold. Engineering carbon nanotubes and nanotube circuits using electrical breakdown. Science, 2001.

[2] A. Bachtold, P. Hadley, T. Nakanishi, and C. Dekker. Logic circuits with carbon nanotube transistors. Science, 294:1317, 2001.

[3] S. Datta. Electronic transport in mesoscopic systems. Cambridge University Press, Cambridge, UK, 1997.

[4] Y. Imry. Introduction to Mesoscopic Physics. Oxford University Press, 1997.

[5] D. K. Ferry and S. M. Goodnick. Transport in nanostructures. Cambridge University Press, 1997.

[6] K. Hansen, E. Laegsgaard, I. Stensgaard, and F. Besenbacher. Quantized conductance in relays. Phys. Rev. B, 56:1022, 1997.

[7] R. P. Andres, T. Bein, M. Dorogi, S. Feng, J. I. Henderson, C. P. Kubiak, W. Mahoney, R. G. Osifchin, and R. Reifenberger. "coulomb staircase" at room temperature in a self-assembled molecular nanostructure. Science, 272:1323, 1996.

[8] M. A. Reed, C. Zhou, C. J. Muller, T. P. Burgin, and J. M. Tour. Conductance of a molecular junction. Science, 278:252, 1997.

[9] C. Kergueris, J.-P. Bourgoin, D. Esteve, C. Urbina, M. Magoga, and C. Joachim. Electron transport through a metal-molecule-metal junction. Phys. Rev. B, 59(19):12505, 1999.

[10] C. Kergueris, J. P. Bourgoin, and S. Palacin. Experimental investigations of the electrical transport properties of dodecanethiol and $\alpha, \omega$ bisthiolterthiophene molecules embedded in metal-molecule-metal junctions. Nanotechnology, 10:8, 1999.

[11] J. Reichert, R. Ochs, H. B. Weber, M. Mayor, and H. v. Löhneysen. Driving current through single organic molecules. cond-mat/0106219, June 2001.

[12] S. Hong, R. Reifenberger, W. Tian, S. Datta, J. Henderson, and C. P. Kubiak. Molecular conductance spectroscopy of conjugated, phenyl-based molecules on au(111): the effect of end groups on molecular conduction. Superlattices and Microstructures, 28:289, 2000.

[13] J. J. W. M. Rosink, M. A. Blauw, L. J. Geerligs, E. van der Drift, and S. Radelaar. Tunneling spectroscopy study and modeling of electron transport in small conjugated azomethine molecules. Phys. Rev. B, 62(15):10459, 2000. 
[14] C. Joachim and J. K. Gimzewski. An electromechanical amplifier using a single molecule. Chem. Phys. Lett., 265:353, 1997.

[15] W. Tian, S. Datta, S. Hong, R. Reifenberger, J. I. Henderson, and P. Kubiak. Conductance spectra of molecular wires. J. Chem. Phys., 109(7):2874, 1998.

[16] J. Chen, W. Wang, M. A. Reed, A. M. Rawlett, D. W. Price, and J. M. Tour. Room-temperature negative differential resistance in nanoscale molecular junctions. Appl. Phys. Lett., 77(8):1224, 2000.

[17] D. Porath, A. Bezryadin, S. de Vries, and C. Dekker. Direct measurement of electrical transport through dna molecules. Nature, 403:635, 2000.

[18] M. Magoga and C. Joachim. Conductance of molecular wires connected or bonded in parallel. Phys. Rev. B, 59(24):16011, 1999.

[19] L. E. Hall, J. R. Reimers, N. S. Hush, and K. Silverbrook. Formalism, analytical model, and a priori green's-function-based calculations of the current-voltage characteristics of molecular wires. J. Chem. Phys., $112: 1510,2000$.

[20] M. Paulsson and S. Stafström. Self-consistent-field study of conduction through conjugated molecules. Phys. Rev. B, 64:035416, 2001.

[21] E. G. Emberly and G. Kirczenow. Multiterminal molecular wire systems: A self-consistent theory and computer simulations of charging and transport. Phys. Rev. B, 62(15):10451, 2000.

[22] P. S. Damle, A. W. Ghosh, and S. Datta. Unified description of molecular conduction: From molecules to metallic wires. Phys. Rev. B, 64:201403(R), 2001.

[23] J. Taylor, H. Gou, and J. Wang. Ab initio modeling of quantum transport properties of molecular electronic devices. Phys. Rev. B, 63:245407, 2001.

[24] M. Di Ventra, S. T. Pantelides, and N. D. Lang. First-principles calculation of transport properties of a molecular device. Phys. Rev. Lett., 84:979, 2000.

[25] P. Damle, A. W. Ghosh, and S. Datta. First-principles analysis of molecular conduction using quantum chemistry software. Preprint.

[26] Y. Q. Xue, S. Datta, and M. A. Ratner. Charge transfer and "band lineup" in molecular electronic devices: A chemical and numerical interpretation. J. Chem. Phys., 115:4292, 2001.

[27] J. J. Palacios, A. J. Pérez-Jiménez, E. Louis, and J. A. Vergés. Fullerenebased molecular nanobridges: A first-principles study. cond-mat/0101359, 2001. 
[28] J. M. Seminario, A. G. Zacarias, and J. M. Tour. Molecular current-voltage characteristics. J. Phys. Chem. A, 1999.

[29] F. Zahid, M. Paulsson, and S. Datta. Advanced Semiconductors and Organic Nano-Techniques, chapter Electrical Conduction through Molecules. Academic press, 2002. Editor H. Markoc, to be published in 2002, for a preprint e-mail 'datta@purdue.edu'.

[30] P. S. Damle, A. W. Ghosh, and S. Datta. Molecular electronics, chapter Theory of nanoscale conduction. 2002. Editor, M. Reed, to be published in 2002, for a preprint e-mail 'datta@purdue.edu'.

[31] S. G. Lias et al. Gas-Phase Ion and Neutral Thermochemistry in Journal of Physical and Chemical Reference Data. American Chemical Society and Americal Insitute of Physics, 1988.

[32] M. C. Desjoqueres and D. Spanjaard. Concepts in Surface Physics. Springer-Verlag, Berlin, Heidelberg, New York, second edition, 1996.

[33] R. G. Parr and W. Yang. Density Functional Theory of Atoms and Molecules, page 99. Oxford University Press, 1989.

[34] J. Tersoff. Theory of semiconductor heterojunctions: The role of quantum dipoles. Phys. Rev. B, 30:4874, 1984.

[35] J. Bardeen. Surface states and rectification at a metal semi-conductor contact. Phys. Rev., 71(10):717, 1947.

[36] V. Mujica, A. E. Roitberg, and M. Ratner. Molecular wire conductance: Electrostatic potential spatial profile. J. Chem. Phys., 112(15):6834, 2000.

[37] Matlab codes used to generate the "toy model" results in Sect. 3 can be obtained by sending email to "datta@purdue.edu".

[38] S. Datta, W. Tian, S. Hong, R. Reifenberger, J. I. Henderson, and C. P. Kubiak. Current-voltage characteristics of self-assembled monolayers by scanning tunneling microscopy. Phys. Rev. Lett., 79:2530, 1997.

[39] A. W. Ghosh et al. Insights from I-V asymmetry in molecular conductors. preprint cond-mat/0202519.

[40] Special Issue on Single Charge Tunneling, volume 85. Z. Phys. B., 1991.

[41] M. Paulsson, F. Zahid, and S. Datta. Nanoscience, Engineering and Technology Handbook, chapter Resistance of a Molecule. CRC Press, 2002. Editors D. Brenner, S. Lyshevski and G. Iafrate, to be published in 2002, for a preprint e-mail 'datta@purdue.edu'.

[42] P. F. Bagwell and T. P. Orlando. Landauer's conductance formula and its generalization to finite voltages. Phys. Rev. B, 40(3):1456, 1989. 
[43] M. Brandbyge, J. Taylor, K. Stokbro, J.-L. Mozos, and P. Ordejon. Density functional method for nonequilibrium electron transport. Preprint condmat/0110650, 2001.

[44] R. Zeller, J. Deutz, and P. Dederichs. Solid State Commun., 44:993, 1982.

[45] R. S. Mulliken, C. A. Rieke, D. Orloff, and H. Orloff. Formulas and numerical tables for overlap integrals. J. Chem. Phys., 17(12):1248, 1949.

[46] R. Hoffman. J. Chem. Phys., 39:1397, 1963.

[47] M. J. Frisch, G. W. Trucks, H. B. Schlegel, G. E. Scuseria, M. A. Robb, J. R. Cheeseman, V. G. Zakrzewski, J. A. Jr. Montgomery, R. E. Stratmann, J. C. Burant, S. Dapprich, J. M. Millam, A. D. Daniels, K. N. Kudin, M. C. Strain, O. Farkas, J. Tomasi, V. Barone, M. Cossi, R. Cammi, B. Mennucci, C. Pomelli, C. Adamo, S. Clifford, J. Ochterski, G. A. Petersson, P. Y. Ayala, Q. Cui, K. Morokuma, D. K. Malick, A. D. Rabuck, K. Raghavachari, J. B. Foresman, J. Cioslowski, J. V. Ortiz, A. G. Baboul, B. B. Stefanov, G. Liu, A. Liashenko, P. Piskorz, I. Komaromi, R. Gomperts, R. L. Martin, D. J. Fox, T. Keith, M. A. Al-Laham, C. Y. Peng, A. Nanayakkara, C. Gonzalez, M. Challacombe, P. M. W. Gill, B. Johnson, W. Chen, M. W. Wong, J. L. Andres, C. Gonzalez, M. Head-Gordon, E. S. Replogle, and J. A. Pople. Gaussian 98, revision a.7. Gaussian, Inc., Pittsburgh PA, 1998.

[48] J. N. Murrell and A. J. Harget. Semi-empirical SCF MO Theory of Molecules. John Wiley \& sons, London, 1972.

[49] See for example, H. Ohnishi, Y. Kondo, and K. Takayanagi. Quantized conductance through individual rows of suspended gold atoms. Nature, 395:780, 1998. A. I. Yanson, G. Rubio Bollinger, H. E. van den Brom, N. Agrait, and J. M. van Ruitenbeek. Formation and manipulation of a metallic wire of single gold atoms. Nature, 395:783, 1998.

[50] See for example, A. Johansson and S. Stafström. Interactions between molecular wires and a gold surface. Chem. Phys. Lett., 322:301, 2000 and references therein.

[51] A. Szabo and N. S. Ostlund. Modern Quantum Chemistry. McGraw-Hill, New York, 1989.

[52] D. Lohez and M. Lannoo. Generalization of the green's-function formalism to nonorthogonal orbitals: Applications to amorphous sio 2 . Phys. Rev. B, 27:5007, 1983 .

[53] M. Samanta. Master's thesis, Purdue University, 1995.

[54] N. W. Ashcroft and N. D. Mermin. Solid state physics, chapter 8. Saunders college publishing, Philadelphia, 1976. Discusses periodic structures and $\mathrm{k}$-space in general. 